\title{
On Gradient Descent Algorithm for Generalized Phase Retrieval Problem
}

\author{
Ji Li, Tie Zhou
}

\begin{abstract}
In this paper, we study the generalized phase retrieval problem: to recover a signal $x \in \mathbb{C}^{n}$ from the measurements $y_{r}=\left|\left\langle\boldsymbol{a}_{r}, \boldsymbol{x}\right\rangle\right|^{2}, r=1,2, \ldots, m$. The problem can be reformulated as a least-squares minimization problem. Although the cost function is nonconvex, the global convergence of gradient descent algorithm from a random initialization is studied, when $m$ is large enough. We improve the known result of the local convergence from a spectral initialization. When the signal $x$ is real-valued, we prove that the cost function is local convex near the solution $\{ \pm x\}$. To accelerate the gradient descent, we review and apply several efficient line search methods. We also perform a comparative numerical study of the line search methods and the alternative projection method. Numerical simulations demonstrate the superior ability of LBFGS algorithm than other algorithms.
\end{abstract}

Index Terms-Phase retrieval, Gradient descent, Global convergence, LBFGS, Local convexity.

\section{INTRODUCTION}

Phase retrieval is to recover a complex signal from its Fourier intensity. This problem arises in many engineering and science applications, such as X-ray crystallography [1], electron microscopy [2], X-ray diffraction imaging [3], optics [4] and astronomy [5], just name a few. In these applications, one often has recorded the Fourier transform intensity of a complex signal, while the phase information is infeasible. Due to the absence of Fourier phase, the available information is incomplete. It has been proved that the one-dimensional phase retrieval problem suffers from essential nonuniqueness, and the multi-dimensional case is usually less prone to multiple solutions [6], [7]. However, those theories did not lead efficient recovery algorithm.

The most widely-used algorithms are based on the method of alternating projections, that are the error reduction (ER) and its variants, such as HIO [5], HPR [8] and RAAR [9]. These iterative projection methods have combined the oversampling method [10] and additional constraints to increase the probability of finding a solution. The forementioned algorithms often work well for real-valued signal in practice and show unsatisfied performance for complex-valued signal [11]. These algorithms have limited recovery abilities due to the issue of convergence to local minimizers. They are identified as the counterparts of iterative projection methods for convexset feasible problem [12]. Since the intensity constraints in

$\mathrm{Ji} \mathrm{Li} \mathrm{is} \mathrm{with} \mathrm{LMAM,} \mathrm{School} \mathrm{of} \mathrm{Mathematical} \mathrm{Sciences,} \mathrm{Peking} \mathrm{University,}$ Beijing 100871, China.

Tie Zhou is with LMAM, School of Mathematical Sciences, Peking University, Beijing 100871, China.

Manuscript received ; revised
Fourier space is not convex, so the algorithms do not have theoretical guarantees.

Recently there has been a renewed interest in phase retrieval due to technological advances in measurement systems and theoretical developments in structured signal recovery, see literature [13] and references therein. In particular, it is now possible to obtain specific kinds of additional intensity information about the signal, depending on the application. The premise of the multiple measurements approach is that, by carefully redesigning the measurement process, one can potentially resolve the phase ambiguity for phase retrieval. Another advantage of this approach is that the analysis and developed algorithms are independent of the dimensional of the signal, as opposed to the alternative projection methods. Mathematically, we consider the generalized phase retrieval problem. It is to find a vector $\boldsymbol{z} \in \mathbb{C}^{n}$, given that

$$
y_{r}=\left|\left\langle\boldsymbol{a}_{r}, \boldsymbol{z}\right\rangle\right|^{2}, \quad r=1,2, \ldots, m
$$

where $\boldsymbol{a}_{r} \in \mathbb{C}^{n}$ are known sampling vectors, and $y_{r} \in \mathbb{R}$ are the intensity measurements. For the recent progress on the generalized phase problem, we refer the reader to the survey papers [3], [14].

The problem (1) can be reformulated as an NP-hard matrix rank minimization problem by lifting a vector to a rank-one matrix. Semi-definite programs (SDP), such as PhaseLift [13], [15] and PhaseCut [16], [17], are used to solve its convex relaxation problem based on two different formulations. PhaseLift is to solve the following convex optimization problem:

$$
\begin{aligned}
\operatorname{minimize} & \operatorname{Tr}(X) \\
\text { s.t. } & \mathcal{A}_{r}(X)=y_{r}, \quad r=1,2, \ldots, m,
\end{aligned}
$$

where $\mathcal{A}_{r}(X)=\operatorname{Tr}\left(\boldsymbol{a}_{r} \boldsymbol{a}_{r}^{*} X\right)=\operatorname{Tr}\left(\boldsymbol{a}_{r} \boldsymbol{a}_{r}^{*} \boldsymbol{x} \boldsymbol{x}^{*}\right)=$ $\left|\left\langle\boldsymbol{a}_{r}, \boldsymbol{x}\right\rangle\right|^{2}=y_{r}$. The main advantage is that the convex optimization has theoretical guarantees and efficient numerical methods. A nature question is how/when the solution of PhaseLift/PhaseCut is also exactly a solution of the original phase problem and how to design the sampling vector to guarantee the recovery. It is shown that the required sampling complexity $m$ is $\mathcal{O}(n)$ for Gaussian model [15] and $\mathcal{O}\left(n \log ^{2} n\right)$ for coded diffraction pattern model [18], [19]. While in principle SDP-based methods offer tractable solution, they become computationally prohibitive as the dimension of the signal increases. So recently, authors of [20] reformulated (11) as a least-squares problem. A solution to the problem (1) 
is any solution to the optimization problem

$$
\underset{\boldsymbol{z} \in \mathbb{C}^{n}}{\operatorname{minimize}} f(\boldsymbol{z}):=\frac{1}{2 m} \sum_{r=1}^{m}\left(\left|\boldsymbol{a}_{r}^{*} \boldsymbol{z}\right|^{2}-y_{r}\right)^{2} .
$$

The fixed-stepsize gradient descent algorithm (called Wirtinger Flow in their paper) is applied to solve (2) in literature [20]. The local convergence to a global minimizer is also shown if the initialization is near the global minimizer.

In this paper, we prove the global convergence of gradient descent algorithm with an appropriate stepsize from a random initialization instead of a good spectral initialization proposed in literature [20]. We find that the algorithm converges to a global minimizer from a random initialization when $m$ is large enough (with the complexity $n \log n$ for Gaussian model and $n \log ^{3} n$ for CDP model) and that all local minimizers of (2) are global minimizers with high probability. In addition, if the signal is real-valued, the local convexity of the leastsquares cost function in 2) is proved. For numerical algorithm, to accelerate the convergence rate, more efficient line search methods for minimizing function of complex variables, such as nonlinear conjugate gradient (NCG) and Limited-memory BFGS (LBFGS), are considered. At the same time of preparing this paper, we find that literature [21] present a geometrical analysis of phase retrieval and apply the trust-region method (TRM) to solve the optimization problem. As opposed to our first-order algorithms, solving a linear equation is needed at each iteration of solving the subproblem of TRM, so our algorithms is better in terms of computational cost.

The remainder of this paper is organized as follows. In Section III, we recall the common least-squares cost function and derive the gradient and Hessian expressions by Fréchet derivative. The expressions are identical to that from the definition of the Wirtinger derivative. It follows gradient descent algorithms with an appropriate stepsize. Besides, some accelerating line search methods are reviewed in Section II-B. In Section III] we present the two main results: one states the convergence to a solution to (1) if provided enough measurements, the other one states the local convexity of the cost function (2) provided the signal is real-valued. In Section IV we test some synthetic models to study the empirical sampling complexity and the empirical performance of the line search methods for solving (1). Section $\mathrm{V}$ concludes the paper with some discussions.

\section{Gradient Descent AND Other First-ORder AlgORITHMS}

We focus on the common least squares cost objective function to measure the misfit between the observed data and predicted data. Recall equation (1), if we denote matrix $A=$ $\left[\boldsymbol{a}_{1}, \boldsymbol{a}_{2}, \ldots, \boldsymbol{a}_{m}\right]^{*} \in \mathbb{C}^{m \times n}$, objective $f(\boldsymbol{z})$ can be rewritten as

$$
f(\boldsymbol{z})=\frac{1}{2 m}\left\||A \boldsymbol{z}|^{2}-\boldsymbol{y}\right\|^{2},
$$

where $|\cdot|$ is the componentwise absolute value, and $\boldsymbol{y}=$ $\left[y_{1}, y_{2}, \ldots, y_{m}\right]^{T}$.

\section{A. Gradient and Hessian}

We derive the gradient and Hessian's analytical expressions of the function $f(z)$ defined by (2) in this subsection. Note that the decision variable $\boldsymbol{z}$ is complex and $f(\boldsymbol{z})$ is realvalued, so the terminologies, gradient and Hessian, can be viewed from the perspective of Wirtinger derivatives [20, see Section 6] or $\mathbb{C}-\mathbb{R}$ calculus [22]. Here we use the Fréchet derivative for an operator defined in Hilbert space to deduce its gradient and Hessian, instead of using the Wirtinger derivative. We find that the expressions resulting from the two approaches are identical and the Fréchet derivative approach is more compact and convenient than Wirtinger derivative approach.

Function $f(\boldsymbol{z})$ (see (3)) can be recognized as an operator defined from Hilbert space $\mathbb{C}^{n}$ to $\mathbb{R}$. Its Fréchet derivative at point $z$ is

$$
D f[\boldsymbol{z}](\boldsymbol{h})=\frac{1}{m}\left\langle D g[\boldsymbol{z}](\boldsymbol{h}),|A \boldsymbol{z}|^{2}-\boldsymbol{y}\right\rangle,
$$

where $g(\boldsymbol{z})=|A \boldsymbol{z}|^{2}-\boldsymbol{y}$. Substituting

$$
D g[\boldsymbol{z}](\boldsymbol{h})=2 \operatorname{Re}(A \boldsymbol{z} \circ \overline{A \boldsymbol{h}})
$$

into (4), we have that

$$
\begin{aligned}
D f[\boldsymbol{z}](\boldsymbol{h}) & =2 m^{-1} \operatorname{Re}\left\langle A \boldsymbol{h} \circ \overline{A \boldsymbol{z}},|A \boldsymbol{z}|^{2}-\boldsymbol{y}\right\rangle \\
& =2 m^{-1} \operatorname{Re}\left\langle A \boldsymbol{h}, A \boldsymbol{z} \circ\left(|A \boldsymbol{z}|^{2}-\boldsymbol{y}\right)\right\rangle \\
& =2 m^{-1} \operatorname{Re}\left\langle\boldsymbol{h}, A^{*}\left(A \boldsymbol{z} \circ\left(|A \boldsymbol{z}|^{2}-\boldsymbol{y}\right)\right)\right\rangle .
\end{aligned}
$$

In a similar way, the Hessian operator (Hessian-vector multiplication) can be derived. Differentiating (6), we get

$$
\begin{aligned}
& D^{2} f[\boldsymbol{z}](\boldsymbol{h}, \boldsymbol{q})=2 m^{-1} \operatorname{Re}\left\langle\overline{A \boldsymbol{q}} \circ A \boldsymbol{h},|A \boldsymbol{z}|^{2}-\boldsymbol{y}\right\rangle \\
&+2 m^{-1} \operatorname{Re}\langle\overline{A \boldsymbol{z}} \circ A \boldsymbol{h}, 2 \operatorname{Re}(A \boldsymbol{z} \circ \overline{A \boldsymbol{q}})\rangle \\
&=2 m^{-1} \operatorname{Re}\left\langle A \boldsymbol{h},\left(2|A \boldsymbol{z}|^{2}-\boldsymbol{y}\right) \circ A \boldsymbol{q}+(A \boldsymbol{z})^{2} \circ \overline{A \boldsymbol{q}}\right\rangle .
\end{aligned}
$$

Let $\boldsymbol{q}=\boldsymbol{h}$, it yields

$$
\begin{aligned}
& D^{2} f[\boldsymbol{z}](\boldsymbol{h}, \boldsymbol{h}) \\
= & 2 m^{-1} \operatorname{Re}\left\langle\boldsymbol{h}, A^{*}\left(\left(2|A \boldsymbol{z}|^{2}-\boldsymbol{y}\right) \circ A \boldsymbol{h}\right)+A^{*}\left((A \boldsymbol{z})^{2} \circ \overline{A \boldsymbol{h}}\right)\right\rangle \\
:=2 \operatorname{Re}\left\langle\boldsymbol{h}, \mathcal{H}_{f}[\boldsymbol{z}](\boldsymbol{h})\right\rangle, & \text { (7) }
\end{aligned}
$$

where $\mathcal{H}_{f}[\boldsymbol{z}](\cdot)$ is the Hessian operator.

According to the Taylor expansion

$$
\begin{gathered}
f(\boldsymbol{z}+\boldsymbol{h})=f(\boldsymbol{z})+D f[\boldsymbol{z}](\boldsymbol{h})+\frac{1}{2} D^{2} f[\boldsymbol{z}](\boldsymbol{h}, \boldsymbol{h})+\text { h.o.t } \\
:=f(\boldsymbol{z})+2 \operatorname{Re}\langle\boldsymbol{h}, \nabla f(\boldsymbol{z})\rangle+\operatorname{Re}\left\langle\boldsymbol{h}, \mathcal{H}_{f}[\boldsymbol{z}](\boldsymbol{h})\right\rangle+\text { h.o.t }
\end{gathered}
$$

so the gradient and Hessian operator are

$$
\begin{aligned}
& \nabla f(\boldsymbol{z})=m^{-1} A^{*}\left(A \boldsymbol{z} \circ\left(|A \boldsymbol{z}|^{2}-\boldsymbol{y}\right)\right) \\
& \mathcal{H}_{f}(\boldsymbol{h})=m^{-1} A^{*}\left(\left(2|A \boldsymbol{z}|^{2}-\boldsymbol{y}\right) \circ A \boldsymbol{h}\right)+A^{*}\left((A \boldsymbol{z})^{2} \circ \overline{A \boldsymbol{h}}\right) .
\end{aligned}
$$

From the definition of Hessian, the Hessian matrix is given by

$$
\nabla^{2} f(\boldsymbol{z})=
$$

$\left[\begin{array}{cc}m^{-1} A^{*} \operatorname{diag}\left(2|A \boldsymbol{z}|^{2}-\boldsymbol{y}\right) A & m^{-1} A^{*} \operatorname{diag}\left((A \boldsymbol{z})^{2}\right) \bar{A} \\ m^{-1} A^{T} \operatorname{diag}\left((\overline{A z})^{2}\right) A & m^{-1} A^{T} \operatorname{diag}\left(2|A \boldsymbol{z}|^{2}-\boldsymbol{y}\right) \bar{A}\end{array}\right]$. 
For easy reference, the gradient and Hessian can be expressed in components $\boldsymbol{a}_{r}$ instead of the above compact form. They have the following forms:

$$
\begin{aligned}
& \nabla f(\boldsymbol{z})=\frac{1}{m} \sum_{r=1}^{m}\left(\left|\boldsymbol{a}_{r}^{*} \boldsymbol{z}\right|^{2}-y_{r}\right)\left(\boldsymbol{a}_{r}^{*} \boldsymbol{z}\right) \boldsymbol{a}_{r}, \\
& \nabla^{2} f(\boldsymbol{z}) \\
& =\frac{1}{m} \sum_{r=1}^{m}\left[\begin{array}{cc}
\left(2\left|\boldsymbol{a}_{r}^{*} \boldsymbol{z}\right|^{2}-y_{r}\right) \boldsymbol{a}_{r} \boldsymbol{a}_{r}^{*} & (10 \mathrm{a}) \\
\left.\left(\overline{\boldsymbol{a}_{r}^{*} \boldsymbol{z}}\right)^{2} \overline{\boldsymbol{a}_{r}} \boldsymbol{a}_{r}^{*} \boldsymbol{z}\right)^{2} \boldsymbol{a}_{r} \boldsymbol{a}_{r}^{T} & \left(2\left|\boldsymbol{a}_{r}^{*} \boldsymbol{z}\right|^{2}-y_{r}\right) \overline{\boldsymbol{a}_{r}} \boldsymbol{a}_{r}^{T}
\end{array}\right] .
\end{aligned}
$$

\section{B. Algorithms: Gradient Descent and Accelerating Strategies}

Sine we have deduced the expression of gradient, we can construct iterative algorithms which are only based on the gradient information. Common optimization algorithms are constructed for real-valued function with real variables. Since the cost function (2) is real-valued with complex variables, the optimization is usually carried out with respect to the real and imaginary part of these variables. Here we consider the straightforward extension of optimization of function of complex variables.

Note that the cost function (2) is nonconvex, so all the line search methods are generally guaranteed to converge to the local minimizers. It is known that Fourier phase retrieval is prone to local minimizer, which is far away from the global minimizer. Using multiple measurements by random masks, we have the advantage that all local minimizers are global minimizers with high probability, see Section III So the line search methods with local convergence, such as nonlinear conjugate gradient (NCG) and limited-memory BFGS (LBFGS), can be applied to the generalized phase retrieval (1) with efficient performance and ensure global convergence in practice.

Line search methods construct a sequence

$$
\boldsymbol{z}_{k+1}=\boldsymbol{z}_{k}+\alpha_{k} \boldsymbol{d}_{k}
$$

The basic idea is first to choose a descent direction $\boldsymbol{d}_{k} \in \mathbb{C}^{n}$, then to refine the iteration with some line search scheme to choose the appropriate step length $\alpha_{k} \in \mathbb{R}$ at $k$ th iteration. The most simple iteration (a.k.a gradient descent) is stated as following: start with an initialization $\boldsymbol{z}_{0} \neq \mathbf{0}$, and inductively update

$$
\boldsymbol{z}_{k+1}=\boldsymbol{z}_{k}-\alpha_{k} \boldsymbol{g}_{k}
$$

where $\alpha_{k}$ is the stepsize and $\boldsymbol{g}_{k}=\nabla f\left(\boldsymbol{z}_{k}\right)$, i.e., taking descent direction $\boldsymbol{d}_{k}=-\boldsymbol{g}_{k}$.

To accelerate the rate of convergence, nonlinear conjugate gradient (NCG) method is widely used. The conjugate gradient direction $\boldsymbol{d}_{k}$ is generated by the recurrence relation

$$
\boldsymbol{d}_{k}=-\boldsymbol{g}_{k}+\beta_{k} \boldsymbol{d}_{k-1},
$$

where $d_{0}=0$. There are a variety of options to choose parameter $\beta_{k}$ for nonlinear problem [23]. In this paper, we take the Hestenes-Stiefel form

$$
\beta_{k}^{H S}=-\frac{\operatorname{Re}\left(\boldsymbol{g}_{k}^{*}\left(\boldsymbol{g}_{k}-\boldsymbol{g}_{k-1}\right)\right)}{\operatorname{Re}\left(\boldsymbol{d}_{k-1}^{*}\left(\boldsymbol{g}_{k}-\boldsymbol{g}_{k-1}\right)\right)} .
$$

Although Newton algorithm has two-order convergence rate near the minimizer, it is not suit for large scale problem, since solving the Newton equation at each iteration is required. We consider the famous LBFGS method, which is appropriate for large-scale problem, and the descent direction $\boldsymbol{d}_{k}$ can be obtained by the easy two-loop recursion, which is described in Algorithm 1 .

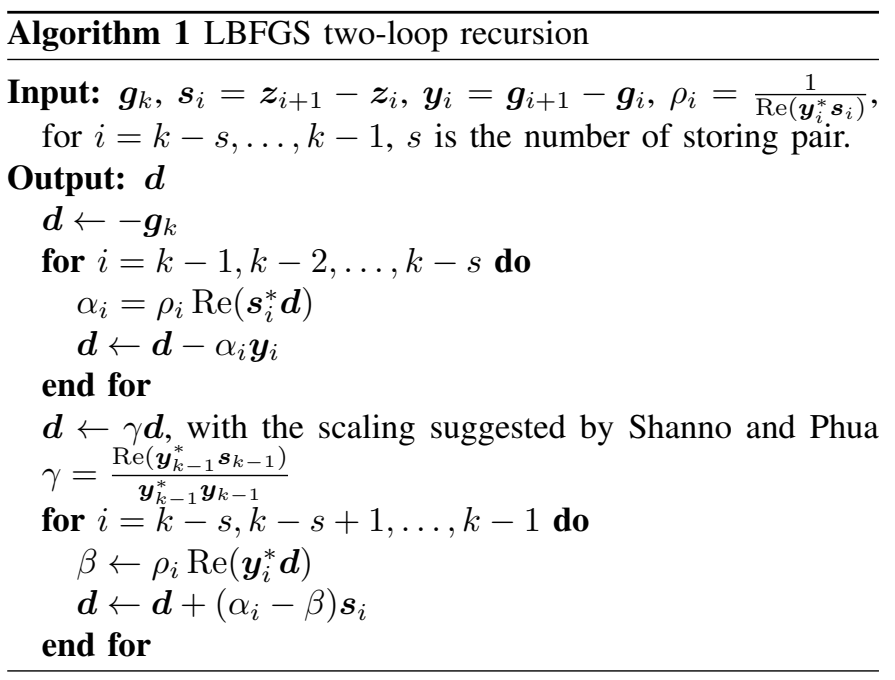

With holding global convergence to local minimizer, step length $\alpha_{k}$ is not arbitrary. For the steepest gradient descent, we strictly characterize the choice strategy of stepsize $\alpha_{k}$, which will lead to global convergence. In next section, we see that our convergence analysis for the gradient descent is based on two idealizations: (i) the solution $\boldsymbol{x}$ is known a priori; and (ii) the stepsize $\alpha_{k}$ is obtained by solving a equation with degree of three, which render the numerical algorithm impractical. For optimization algorithms, there is usually a chosen strategy of $\alpha_{k}$, which are known as Wolfe conditions:

$$
f\left(\boldsymbol{z}_{k}+\alpha_{k} \boldsymbol{d}_{k}\right) \leq f\left(\boldsymbol{z}_{k}\right)+c_{1} \alpha_{k} \operatorname{Re}\left(\boldsymbol{d}_{k}^{*} \boldsymbol{g}_{k}\right)
$$

and

$$
\operatorname{Re}\left(\boldsymbol{d}_{k}^{*} \boldsymbol{g}_{k+1}\right) \geq c_{2} \operatorname{Re}\left(\boldsymbol{d}_{k}^{*} \boldsymbol{g}_{k}\right)
$$

where condition $0<c_{1}<c_{2}<1$ is satisfied. Generally we take $c_{1}=10^{-4}, c_{2}=0.9$ as commended in book [24]. Equations (14a) and (14b) are known as the sufficient decrease and curvature condition respectively. We call gradient descent with stepsize $\alpha_{k}$ by Wolfe conditions steepest gradient (SD) algorithm.

Our theoretical results about global convergence is only applied to the simple gradient descent algorithm. But as we will see in Section IV the numerical performance of NCG and LBFGS is superior than the steepest descent (a.k.a, gradient descent with line search scheme by Wolfe conditions), even the gradient descent we consider here. The complexities of line search scheme and the choice of descent direction of NCG and LBFGS hinder the analysis of the convergence. 


\section{THEORETICAL RESULTS}

We assume that the sampling vectors of the model setup are in the Gaussian [15] or coded diffraction pattern (CDP) [18], [20] models, which are defined below. Gaussian model has more theoretical interests than CDP, but the latter is a more physical realizable model.

If $\boldsymbol{a}_{r} \in \mathbb{C}^{n}$ are drawn from $N(\mathbf{0}, \boldsymbol{I} / 2)+i N(\mathbf{0}, \boldsymbol{I} / 2)$, we say that sampling vectors follow the Gaussian model. In CDP model, we collect multiple diffraction patterns with different masks $\boldsymbol{d}_{l} \in \mathbb{C}^{n}$. The observation data are

$$
\begin{aligned}
y_{l, k}=\left|\left\langle\boldsymbol{f}_{k}, \boldsymbol{d}_{l} \circ \boldsymbol{x}\right\rangle\right|^{2}=\left|\boldsymbol{f}_{k}^{*} D_{l} \boldsymbol{x}\right|^{2}, \\
\quad l=1,2, \ldots, L, \quad k=1,2, \ldots, n, \quad D_{l}=\operatorname{diag}\left(\boldsymbol{b}_{l}\right),
\end{aligned}
$$

where $\boldsymbol{f}_{k}^{*}$ is the $k$ th row of the discrete Fourier transform matrix, i.e., $\boldsymbol{f}_{k}=[1, \exp (i 2 \pi(k-1) / n), \ldots, \exp (i 2 \pi(n-$ $1)(k-1) / n)]^{T}$. And each entry of mask $\boldsymbol{b}_{l}$ samples from a distribution $b$. Assume the entry $b=b_{1} b_{2}$, where $b_{1}$ and $b_{2}$ are independent and distributed as: $b_{1}$ is sampled from $\{-1,1,-i, i\}$ with equal probability $1 / 4$, and $b_{2}$ from $\{\sqrt{2} / 2, \sqrt{3}\}$ with probability $4 / 5$ and $1 / 5$ respectively. This pattern is called octanary in paper [20].

We present the main results in this section and put the proofs and technical lemmas in the Appendix of this paper.

\section{A. Global Convergence of Gradient Descent}

Definition 1. Let $\boldsymbol{x} \in \mathbb{C}^{n}$ be any solution to the phase retrieval problem (1). For each $z \in \mathbb{C}^{n}$, define

$$
\phi(\boldsymbol{z})=\underset{\phi \in[0,2 \pi]}{\arg \min }\left\|\boldsymbol{z}-e^{i \phi} \boldsymbol{x}\right\|, \quad \boldsymbol{h}(\boldsymbol{z})=\frac{\boldsymbol{z}-e^{i \phi(\boldsymbol{z})} \boldsymbol{x}}{\left\|\boldsymbol{z}-e^{i \phi(\boldsymbol{z})} \boldsymbol{x}\right\|},
$$

then the distance of $\boldsymbol{z}$ to the solution set $\chi=\left\{\boldsymbol{x} e^{i \theta}: \theta \in\right.$ $[0,2 \pi)\}$ is

$$
\operatorname{dist}(\boldsymbol{z}, \boldsymbol{x}):=\operatorname{dist}(\boldsymbol{z}, \chi)=\left\|\boldsymbol{z}-\boldsymbol{x} e^{i \phi(\boldsymbol{z})}\right\| .
$$

For real case,

$$
\operatorname{dist}(\boldsymbol{z}, \boldsymbol{x}):=\operatorname{dist}(\boldsymbol{z}, \chi)=\min \{\|\boldsymbol{z}-\boldsymbol{x}\|,\|\boldsymbol{z}+\boldsymbol{x}\|\} .
$$

It is not difficult to see that $\operatorname{Im}\left(\boldsymbol{h}^{*} \boldsymbol{x} e^{i \phi(\boldsymbol{z})}\right)=0$ and $\operatorname{Re}\left(\boldsymbol{h}^{*} \boldsymbol{x} e^{i \phi(\boldsymbol{z})}\right)=\|\boldsymbol{x}\|$.

Lemma III.1 ([20], Lemma 7.1). Assume that the solution $\boldsymbol{x}$ of the phase retrieval problem is independent from the sampling vectors. Furthermore, the sampling vectors $\boldsymbol{a}_{r}$ are distributed according to either the Gaussian or admissible CDP model. Then

$$
\begin{aligned}
& \mathbb{E}\left[\nabla^{2} f(\boldsymbol{x})\right]=\left[\begin{array}{cc}
\|\boldsymbol{x}\|^{2} I+\boldsymbol{x} \boldsymbol{x}^{*} & 2 \boldsymbol{x} \boldsymbol{x}^{T} \\
2 \overline{\boldsymbol{x}} \boldsymbol{x}^{*} & \|\boldsymbol{x}\|^{2} I+\overline{\boldsymbol{x}} \boldsymbol{x}^{T}
\end{array}\right] \\
& =\|x\|^{2} I+\frac{3}{2}\left[\begin{array}{l}
\boldsymbol{x} \\
\overline{\boldsymbol{x}}
\end{array}\right]\left[\begin{array}{ll}
\boldsymbol{x}^{*} & \boldsymbol{x}^{T}
\end{array}\right]-\frac{1}{2}\left[\begin{array}{c}
\boldsymbol{x} \\
-\overline{\boldsymbol{x}}
\end{array}\right]\left[\begin{array}{ll}
\boldsymbol{x}^{*} & -\boldsymbol{x}^{T}
\end{array}\right] .
\end{aligned}
$$

Lemma III.2. For the expectation of the Hessian $\mathbb{E}\left[\nabla^{2} f(\boldsymbol{x})\right]$, then its eigenvalues are $\{4\|\boldsymbol{x}\|^{2}, \underbrace{\|\boldsymbol{x}\|^{2}, \ldots,\|\boldsymbol{x}\|^{2}}_{2 n-2 \text { terms }}, 0\}$. Furthermore the expectation matrix is semi-definite. In the real case, i.e., the solution $x \in \mathbb{R}^{n}$, the expectation of the Hessian $\mathbb{E}\left[\nabla^{2} f(\boldsymbol{x})\right]$ is $\|\boldsymbol{x}\|^{2} I+3 \boldsymbol{x} \boldsymbol{x}^{T}$. It is definite, since its eigenvalues are $\{4\|\boldsymbol{x}\|^{2}, \underbrace{\|\boldsymbol{x}\|^{2}, \ldots,\|\boldsymbol{x}\|^{2}}_{n-1 \text { terms }}\}$.

Remark. Obviously, vector $\left[\begin{array}{l}\boldsymbol{x} \\ \overline{\boldsymbol{x}}\end{array}\right]$ and $\left[\begin{array}{c}i \boldsymbol{x} \\ -i \overline{\boldsymbol{x}}\end{array}\right]$ are the eigenvectors corresponding to eignevalues $4\|\boldsymbol{x}\|^{2}$ and 0 respectively by calculus.

Lemma III.3 ([20], Lemma 7.4). Assume the vectors $\boldsymbol{a}_{r}$ are distributed according to either the Gaussian or admissible CDP model with a sufficiently large number of measurements. This means that the number of samples obeys $m \geq c(\delta) n \log n$ in the Gaussian model and the number of patterns obeys $L \geq c(\delta) \log ^{3} n$ in the CDP model. Then

$$
\left\|\nabla^{2} f(\boldsymbol{x})-\mathbb{E}\left[\nabla^{2} f(\boldsymbol{x})\right]\right\| \leq \delta\|\boldsymbol{x}\|^{2}
$$

holds with probability at least $1-10 e^{-\gamma n}-8 / n^{2}$ and $1-$ $(2 L+1) / n^{3}$ for the Gaussian and CDP models respectively.

Lemma III.4. Let $z \in \mathbb{C}^{n}$ be a fixed vector independent of the sampling vectors. We have

$$
\mathbb{E}[\nabla f(\boldsymbol{z})]=\left(2\|\boldsymbol{z}\|^{2}-\|\boldsymbol{x}\|^{2}\right) \boldsymbol{z}-\left(\boldsymbol{x}^{*} \boldsymbol{z}\right) \boldsymbol{x} .
$$

Lemma III.5. In the setup of Lemma III.3, let $\boldsymbol{z} \in \mathbb{C}^{n}$ be a fixed vector independent of the sampling vectors. Then

$$
\|\nabla f(\boldsymbol{z})-\mathbb{E}[\nabla f(\boldsymbol{z})]\| \leq \frac{\delta}{2}\|\mathbb{E}[\nabla f(\boldsymbol{z})]\|
$$

holds with probability at least $1-20 e^{-\gamma m}-4 m / n^{4}$ in the Gaussian model and $1-(4 L+2) / n^{3}$ in the CDP model. Furthermore, if $\boldsymbol{z}$ obeying $\operatorname{dist}(\boldsymbol{z}, \boldsymbol{x}) \leq\|\boldsymbol{x}\| / 2$, then

$$
\|\nabla f(\boldsymbol{z})-\mathbb{E}[\nabla f(\boldsymbol{z})]\| \leq 4 \delta \operatorname{dist}(\boldsymbol{z}, \boldsymbol{x})\|\boldsymbol{x}\|^{2}
$$

holds with the same high probability.

Lemma III.6. In the setup of LemmaIII.3 then $\mathbb{E}[\nabla f(\boldsymbol{z})]$ and $\boldsymbol{h}$ share the same direction and $\mathbb{E}[\nabla f(\boldsymbol{z})]=\mathbf{0}$ if and only if $z$ is a solution of the phase retrieval problem (1), which holds with high probability. Furthermore, the angle between $\nabla f(\boldsymbol{z})$ and $\boldsymbol{z}-\boldsymbol{x} e^{i \phi(\boldsymbol{z})}$ is below $\arcsin (\delta / 2)$, i.e., the following

$\operatorname{Re}\left\langle\nabla f(\boldsymbol{z}), \boldsymbol{z}-\boldsymbol{x} e^{i \phi(\boldsymbol{z})}\right\rangle \geq \sqrt{1-\frac{\delta^{2}}{4}}\|\nabla f(\boldsymbol{z})\|\left\|\boldsymbol{z}-\boldsymbol{x} e^{i \phi(\boldsymbol{z})}\right\|$

holds with high probability.

Remark. From Lemma III.6, $\nabla f(\boldsymbol{z})=\mathbf{0}$ if and only if $\boldsymbol{z}=\mathbf{0}$ or $z$ is a solution of the phase retrieval problem (1) with high probability. So all local minimizers are global minimizers with high probability, which facilitates the line search methods.

Theorem III.7 (Global Convergence). Let $\boldsymbol{x}$ be a solution to the generalized phase retrieval problem (1) and the number of samples $m$ obeys $m \geq c_{1}(\delta) n \log n$ in Gaussian model or the number of patterns obeys $L \geq c_{2}(\delta) \log ^{3} n$ in the $C D P$ model, where $c_{1}, c_{2}$ are sufficiently large numerical constants. For the following gradient descent updating scheme $\left(z_{0} \neq 0\right)$

$$
\boldsymbol{z}_{k+1}=\boldsymbol{z}_{k}-\alpha_{k} \nabla f\left(\boldsymbol{z}_{k}\right)
$$


the (strict) descent property

$$
\operatorname{dist}\left(\boldsymbol{z}_{k+1}, \boldsymbol{x}\right)<\operatorname{dist}\left(\boldsymbol{z}_{k}, \boldsymbol{x}\right)
$$

holds with probability at least $1-20 e^{-\gamma m}-4 m / n^{4}$ in Gaussian model and $1-(4 L+2) / n^{3}$ in CDP model, if the stepsize $\alpha_{k}$ satisfies

$$
\begin{aligned}
& 0<\alpha_{k} \\
& <\min \left\{2 \sqrt{1-\frac{\delta^{2}}{4}} \frac{\left\|\nabla f\left(\boldsymbol{z}_{k}\right)\right\|}{6(2+\delta)\|\boldsymbol{x}\|^{2}}, 2 \sqrt{1-\frac{\delta^{2}}{4}} \sqrt[3]{\frac{\left\|\nabla f\left(\boldsymbol{z}_{k}\right)\right\|}{6(2+\delta)}}\right\} .
\end{aligned}
$$

Furthermore, we denote the intersection of the polynomial of degree three $P_{1}(t)=(2+\delta) t\left(t^{2}+3 t\|\boldsymbol{x}\|+2\|\boldsymbol{x}\|^{2}\right)$ and $y=\left\|\nabla f\left(z_{k}\right)\right\|$ as $t_{1}$ and take stepsize $\alpha_{k}=\sqrt{1-\delta^{2} / 4} t_{1}$, then the geometrical convergence is ensured, that is

$$
\operatorname{dist}\left(\boldsymbol{z}_{k+1}, \boldsymbol{x}\right) \leq\left(\frac{2 \sqrt{2} \delta}{2-\delta}+\frac{\delta}{\sqrt{2}}\right) \operatorname{dist}\left(\boldsymbol{z}_{k}, \boldsymbol{x}\right) .
$$

It is inconvenient to determine $\alpha_{k}$ by finding a root of function, we can relax the $\alpha_{k}$ in two cases.

Corollary III.8. In the setup of Theorem III.7 if $\operatorname{dist}\left(\boldsymbol{z}_{\boldsymbol{k}}, \boldsymbol{x}\right) \leq\|\boldsymbol{x}\| / 5$, then $\alpha_{k}$ can be taken as $\sqrt{1-\delta^{2} / 4} \frac{25\left\|\nabla f\left(\boldsymbol{z}_{k}\right)\right\|}{66(2+\delta)\|\boldsymbol{x}\|^{2}}$. we have

$$
\operatorname{dist}\left(\boldsymbol{z}_{k+1}, \boldsymbol{x}\right) \leq\left(\frac{\sqrt{2}(58 \delta+16)}{25(2-\delta)}+\frac{\delta}{\sqrt{2}}\right) \operatorname{dist}\left(\boldsymbol{z}_{k}, \boldsymbol{x}\right) ;
$$

If $\operatorname{dist}\left(\boldsymbol{z}_{\boldsymbol{k}}, \boldsymbol{x}\right) \geq 2\|\boldsymbol{x}\|$, and $\alpha_{k}=\sqrt{1-\delta^{2} / 4} \sqrt[3]{\frac{\left\|\nabla f\left(\boldsymbol{z}_{k}\right)\right\|}{3(2+\delta)}}$, we have

$$
\operatorname{dist}\left(\boldsymbol{z}_{k+1}, \boldsymbol{x}\right) \leq\left(\frac{\sqrt{2}(4 \delta+4)}{3(2-\delta)}+\frac{\delta}{\sqrt{2}}\right) \operatorname{dist}\left(\boldsymbol{z}_{k}, \boldsymbol{x}\right) .
$$

Remark. 1) Theorem III.7 tells us that the gradient descent algorithm with appropriate stepsize converges to a solution of (1) when given enough many measurements. It is an extension of the local convergence of gradient descent method, see [20, Theorem 3.3]. Our proof is more geometrical than the proof in literature [20].

2) From the Lemma III.6, for any nonzero vector $z \in \mathbb{C}^{n}$ outside the solution set $\chi, \nabla f(\boldsymbol{z}) \neq 0$ hold with probability at least $1-20 e^{-\gamma m}-4 m / n^{4}$ in Gaussian model and $1-(4 L+2) / n^{3}$ in CDP model. Thus, there is no saddle point of $f(\boldsymbol{z})$ with high probability. When $m$ is large enough, $\left\{\boldsymbol{x} e^{i \theta}\right\}$ are the only local minimizers, and also global minimizers. This fact makes the optimization approach easily to find a solution, it is also noticed by authors of literature [25].

3) As opposed to Wirtinger Flow (WF) algorithm, we do iterate from a random initialization without a careful choice of initialization. The algorithm looses at most a logarithmic factor in the sampling complexity as the WF. It is an open question whether the complexity can be proportional to $n$.

4) Although the norm of solution $\|\boldsymbol{x}\|$ is not known a priori, it has an uniform upper bound with high probability in Gaussian model, since we have $\mathbb{E}\left[\left|\boldsymbol{a}_{r}^{*} \boldsymbol{x}\right|^{2}\right]=\|\boldsymbol{x}\|^{2}$.
It is known that the phase retrieval problem is less difficult to solve when the target signal $\boldsymbol{x}$ is real. We shall give the theoretical aspect why it happens. It is due to the local convexity of $f(\boldsymbol{z})$ when $\boldsymbol{z}$ is in a neighborhood of the solution $\boldsymbol{x}$.

\section{B. Local Convexity with Real-Valued Signal}

We assume the target signal $\boldsymbol{x} \in \mathbb{R}^{n}$ and the sampling vectors $\boldsymbol{a}_{r}$ are drawn from the Gaussian model. The gradient and Hessian of objective $f$ have the following form (up to a scale factor $1 / 2)$ :

$$
\begin{aligned}
\nabla f(\boldsymbol{z}) & =\frac{1}{m} \sum_{r=1}^{m} \operatorname{Re}\left(\left(\left|\boldsymbol{a}_{r}^{*} \boldsymbol{z}\right|^{2}-y_{r}\right)\left(\boldsymbol{a}_{r}^{*} \boldsymbol{z}\right) \boldsymbol{a}_{r}\right), \\
\nabla^{2} f(\boldsymbol{z}) & =\frac{1}{m} \sum_{r=1}^{m} \operatorname{Re}\left(\left(2\left|\boldsymbol{a}_{r}^{*} \boldsymbol{z}\right|^{2}-y_{r}\right) \boldsymbol{a}_{r} \boldsymbol{a}_{r}^{*}+\left(\boldsymbol{a}_{r}^{*} \boldsymbol{z}\right)^{2} \boldsymbol{a}_{r} \boldsymbol{a}_{r}^{T}\right) .
\end{aligned}
$$

Lemma III.9. Let $z \in \mathbb{R}^{n}$ be a fixed vector independent of the sampling vectors. Then we have

$$
\begin{aligned}
\mathbb{E}\left[\nabla^{2} f(\boldsymbol{z})\right] & =\left[\begin{array}{cc}
A & 2 \boldsymbol{z} \boldsymbol{z}^{T} \\
2 \overline{\boldsymbol{z}} \boldsymbol{z}^{*} & \bar{A}
\end{array}\right], \\
\text { with } A & =\left(2\|\boldsymbol{z}\|^{2}-\|\boldsymbol{x}\|^{2}\right) I+2 \boldsymbol{z} \boldsymbol{z}^{*}-\boldsymbol{x} \boldsymbol{x}^{*} .
\end{aligned}
$$

For real case, the expectation matrix for a fixed vector $z$,

$$
\mathbb{E}\left[\nabla^{2} f(\boldsymbol{z})\right]=\left(2\|\boldsymbol{z}\|^{2}-\|\boldsymbol{x}\|^{2}\right) I+4 \boldsymbol{z} \boldsymbol{z}^{T}-\boldsymbol{x} \boldsymbol{x}^{T} .
$$

Lemma III.10 ([20], Lemma 7.3). Assume $\boldsymbol{u}, \boldsymbol{v} \in \mathbb{C}^{n}$ are fixed vectors obeying $\|\boldsymbol{u}\|=\|\boldsymbol{v}\|=1$ which are independent of the sampling vectors. Furthermore, assume the measurement vectors $\boldsymbol{a}_{r}$ are distributed according to the Gaussian model. Then

$$
\begin{aligned}
\mathbb{E}\left[\left|\boldsymbol{a}_{r}^{*} \boldsymbol{u}\right|^{2 k}\right] & =k ! \\
\mathbb{E}\left[\operatorname{Re}\left(\boldsymbol{u}^{*} \boldsymbol{a}_{r} \boldsymbol{a}_{r}^{*} \boldsymbol{v}\right)\left|\boldsymbol{a}_{r}^{*} \boldsymbol{u}\right|^{2}\right] & =2 \operatorname{Re}\left(\boldsymbol{u}^{*} \boldsymbol{v}\right) \\
\mathbb{E}\left[\left(\operatorname{Re}\left(\boldsymbol{u}^{*} \boldsymbol{a}_{r} \boldsymbol{a}_{r}^{*} \boldsymbol{v}\right)\right)^{2}\right] & =\frac{1}{2}+\frac{3}{2}\left(\operatorname{Re}\left(\boldsymbol{u}^{*} \boldsymbol{v}\right)\right)^{2}-\frac{1}{2}\left(\operatorname{Im}\left(\boldsymbol{u}^{*} \boldsymbol{v}\right)\right)^{2} .
\end{aligned}
$$

Theorem III.11 (Convexity on Expectation (Asymptotic Convexity)). Let $z \in \mathbb{R}^{n}$ be a fixed vector independent of the sampling vectors, we have that $\mathbb{E}[f(\boldsymbol{z})]$ is convex in the ellipse $\operatorname{dist}(\boldsymbol{z}, \boldsymbol{x}) \leq\|\boldsymbol{x}\| / 12$.

In this proof, we use the following fact that the eigenvalues of matrix $\left(\boldsymbol{x}^{T} \boldsymbol{w}\right) I+\boldsymbol{x} \boldsymbol{w}^{T}+\boldsymbol{w} \boldsymbol{x}^{T}$ are

$$
\{\underbrace{\boldsymbol{x}^{T} \boldsymbol{w}, \ldots, \boldsymbol{x}^{T} \boldsymbol{w}}_{n-2 \text { terms }}, 2 \boldsymbol{x}^{T} \boldsymbol{w} \pm\|\boldsymbol{x}\|\} .
$$

It can be obtained from the Lemma A.1.

Theorem III.12 (Strong Convex). In the setup of Theorem III.7 for all $\boldsymbol{z} \in \mathbb{R}^{n}$ in the ellipse around $\boldsymbol{x}$, more specified, $\operatorname{dist}(\boldsymbol{z}, \boldsymbol{x}) \leq\|\boldsymbol{x}\| / 24$, the following

$$
\boldsymbol{w}^{T} \nabla^{2} f(\boldsymbol{z}) \boldsymbol{w} \geq 0
$$

holds uniformly with probability $1-e^{-\alpha n}$ ( $\alpha$ depends on $m$ ), where $\boldsymbol{w} \in \mathbb{R}^{n}$ such that $\|\boldsymbol{w}\|=1$. 
Remark. Since the proof of local convexity is based on the Lemma III.10, the result holds only for sampling vectors followed Gaussian model. It is an open problem whether it holds for CDP model or not.

From the analysis above, we find that the randomness assumption is useful for theoretical analysis. Theorems III.11 and III.12 state the convexity of the expectation of $f(\boldsymbol{z})$ and the strong convexity of function $f(\boldsymbol{z})$ when $\boldsymbol{z}$ is around a solution of the phase retrieval problem. These results imply the numerical algorithms will perform well in the neighborhood of a solution. And the local geometrical convergence rate is followed from the convex optimization.

\section{NUMERICAL SimUlations}

This section introduces numerical simulations to illustrate the empirical sampling complexity to ensure the convergence to a solution of the optimization approach and study the effectiveness of line search methods, especially the LBFGS method. Since gradient descent with stepsize $\alpha_{k}$ from Theorem III.7 takes more iterations than that with stepsize $\alpha_{k}$ from Wolfe conditions. For comparison, we only consider the steepest gradient descent algorithm.

\section{A. Relative Error and Termination Condition}

We denote the solution to the problem (1) as $\boldsymbol{x}$, and $\boldsymbol{z}$ is the returned solution by the line search method, the relative error is defined as

$$
\text { relerr }=\operatorname{minimize}_{|c|=1} \frac{\|c \boldsymbol{x}-\boldsymbol{z}\|_{2}}{\|\boldsymbol{x}\|_{2}},
$$

where $c$ is to get rid of the effect of the constant phase shift of phase problem. From

$$
\langle c \boldsymbol{x}-\boldsymbol{z}, c \boldsymbol{x}-\boldsymbol{z}\rangle=|c|^{2}\|\boldsymbol{x}\|_{2}^{2}-2 \operatorname{Re}\langle c \boldsymbol{x}, \boldsymbol{z}\rangle+\|\boldsymbol{z}\|_{2}^{2},
$$

the constant $c$ is given by

$$
c=\frac{\langle\boldsymbol{x}, \boldsymbol{z}\rangle}{|\langle\boldsymbol{x}, \boldsymbol{z}\rangle|} .
$$

We consider a vector to be successfully recovered if the relative error is below $10^{-5}$.

In line search methods, for stop criteria, we take the three options: the number of iterations is 600 , the tolerances for relative change of objective function and relative change of variable during one iteration both are $10^{-12}$. The number of stored vector pairs for LBFGS method $s=2$.

\section{B. Recovery Rate}

We begin by examining the empirical recovery rate of the line search method (LBFGS is used) for recovering random Gaussian signal $\boldsymbol{x} \in \mathbb{R}^{n}$ or $\mathbb{C}^{n}$ under the Gaussian and CDP models with octanary pattern. In the one dimensional simulations, we consider signals with length $n=512,1014$. For two dimensional tests, the test signals are in size $n=$ $n_{1} \times n_{2}=128 \times 128$. All test signals are drawn from the Gaussian distribution, that is $\boldsymbol{x} \sim N(0, I)$ when $\boldsymbol{x} \in \mathbb{R}^{n}$ and $\boldsymbol{x} \sim N(0, I)+i N(0, I)$ when $\boldsymbol{x} \in \mathbb{C}^{n}$. The algorithm is tested for 17 values of $m=\delta n$, where $\delta=2,2.5, \ldots, 9.5,10$ for Gaussian model. In CDP model, algorithm is tested for 9 values of $L$ from 2 to 10 . We report the empirical probability of success in the two models in Figure 11. The empirical probability of success is an average over 1000 trials, we generate 10 different test signals and corresponding random sampling matrix $A$ in Gaussian model or random masks in CDP model and begin the algorithm from 100 random initials for a fixed random sampling matrix.

No matter what the sampling vectors are, in Gaussian model or CDP model, for a signal with length $n=1024$, the optimization method can successfully recover the signal when $m=7 n$, see Figure $1 \mathrm{a}$. Note that $m$ is the order $n \log n$, which matches our analysis. This empirical sampling complexity is greater than that of WF with spectral initialization, WF can recover the signal when $m=4 n$ [20]. It is not surprising for this fact, the need for more sampling vectors is at the price of beginning from a random initialization.

Assume the signal is real and the sampling vectors are in CDP model, the algorithm recovers the real signals with sampling patterns $L=5$. It recovers the real signals with sampling patterns $L=8$ without the priori constraint of realvaluedness. Figure $1 \mathrm{~b}$ illustrate this observation.

\section{Alternative Projection}

For comparison, we also apply the alternative projection algorithm to phase retrieval problem. In the setting of multiple illuminations, the algorithm is described in Algorithm 2

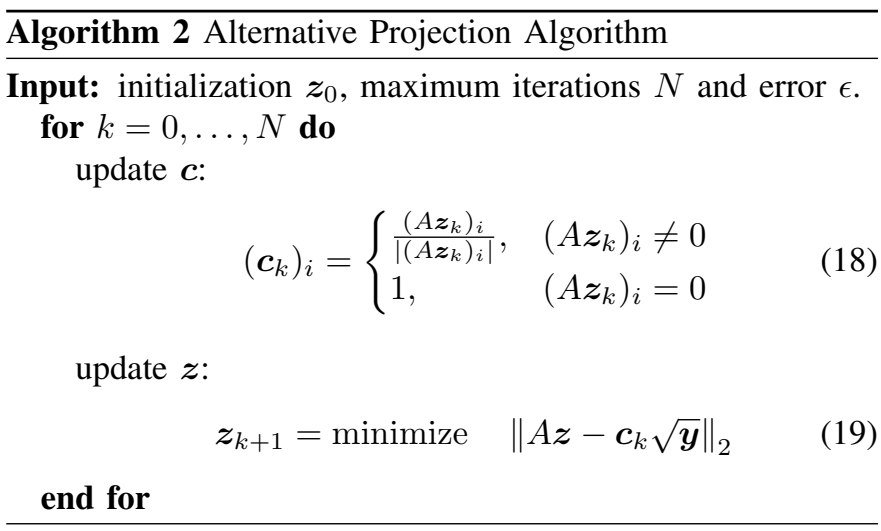

where solution $\boldsymbol{z}_{k+1}$ to 19 is $A^{\dagger}\left(\boldsymbol{c}_{\boldsymbol{k}} \sqrt{\boldsymbol{y}}\right)$, where

$$
A^{\dagger}\left(\begin{array}{c}
\boldsymbol{\omega}_{1} \\
\vdots \\
\boldsymbol{\omega}_{L}
\end{array}\right)=\frac{1}{\sum_{l}\left|\boldsymbol{d}_{l}\right|^{2}}\left(\sum_{l=1}^{L} \overline{\boldsymbol{d}}_{l} \cdot F^{*}\left(\boldsymbol{\omega}_{l}\right)\right) \text {. }
$$

The alternative projection method converges geometrically to a solution of problem (1) under the Gaussian model with large enough measurements, this fact is proven in literature [26]. If we apply it from a random initialization, its overall 


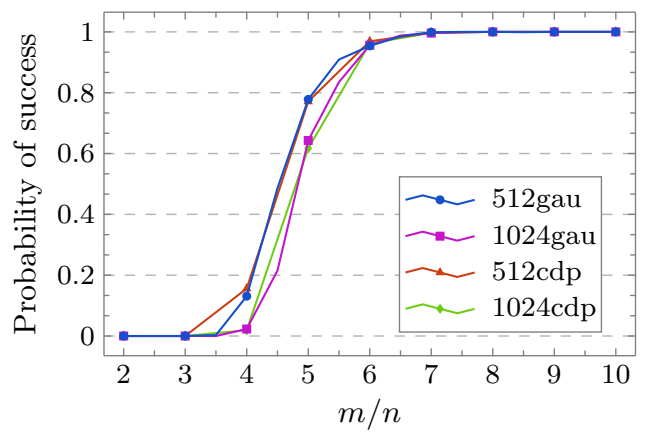

(a) Recovery probability for 1-D signals in Gaussian and CDP models

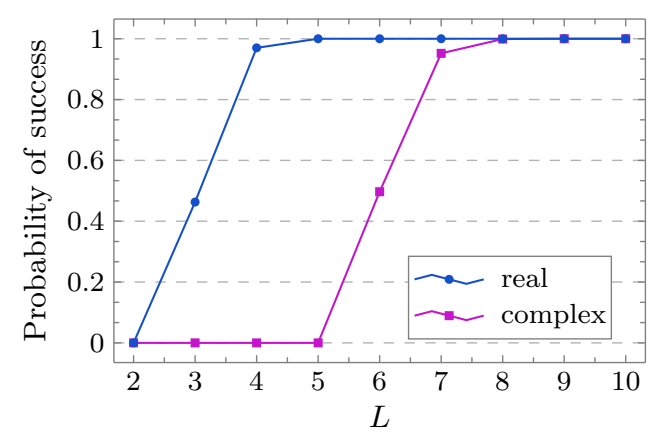

(b) Recovery probability for 2-D signals in CDP model

Fig. 1: Average recovery probability

performance is worse than optimization approach. Figure 5 illustrates this phenomenon.

\section{Performance for the CDP Model}

The test images is a complex-valued image of size $512 \times 512$, whose pixel values correspond to the complex transmission coefficients of a collection of gold balls embedded in a medium. Its magnitude is shown in Figure 2a We only consider the CDP model, the stylized setup of coded diffraction pattern, which one encounters in X-ray crystallography and many other imaging sciences.

1) Noise-free and Noise Measurements: In the first experiment, we demonstrate the recovery of the image from noiseless measurements. We consider two different types of illuminations. The first type uses ten octanary masks. The reconstruction is shown in Figure 2b, It is visually indistinguishable from the original image. Since they are both complex-valued, we display only the magnitude. We also achieve successful recovery with eight octanary masks.

Octanary masks may not be realizable in practice. Our second example uses simple random binary masks, where the entries are either 0 or 1 with equal probability. In this case, a large number of illuminations are required to achieve a reconstruction of comparable quality. The result for ten binary illuminations, one being regular Fourier measurement, is shown in Figure 2c.

In the second set of experiments we consider the same test image but with noisy measurements. Ten octanary masks as before are used. Since the main noise yields Poisson distribution resulting from the photon counting in practice, we add random Poisson noise to the measurements for ten different SNR levels, ranging from $10 \mathrm{~dB}$ to $55 \mathrm{~dB}$. Figure 3 shows the reconstructions from two SNR level data. Figure 3a and $3 \mathrm{~b}$ depict the resulting reconstructions for low SNR case $(10 \mathrm{~dB})$ and high SNR case $(30 \mathrm{~dB})$, respectively. Figure 4 shows the average relative error in $\mathrm{dB}$ versus the SNR. The error curves shows clearly the linear behavior between SNR and relative error, it implies the stability of the generalized phase retrieval problem [15].

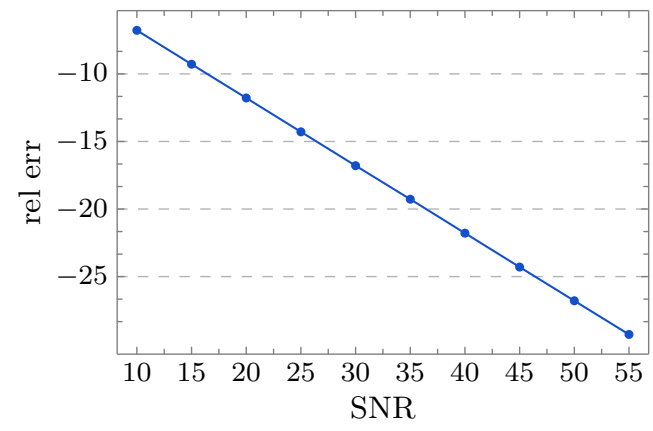

Fig. 4: Relative error in $\mathrm{dB}$ vs SNR

2) Performance Comparison of Different Algorithms: We study the performance of different line search method (SD, NCG and LBFGS) and alternative projection method (AP). It shows the effectiveness of the LBFGS to apply to the optimization problem.

Figure 5 a depicts the relation between the relative error and different methods. When the relative error is below $1 e-5$, then the algorithms break. The alternative projection (AP) decrease slowly at the beginning 500 iterations, when it is near the neighborhood of the solution, it decreases fast. For line search methods, the nonlinear conjugate gradient (NCG) and LBFGS have the competitive performance. In terms of the number of iterations, NCG takes fewer iterations than LBFGS. The overall calls of FFT are listed in Table II The LBFGS algorithm need the fewest computations. So the LBFGS performs best for the problem. This observation is the same as the authors' previous paper. It is shown that optimization approach is more robust than alternative projection, where in 10 runs, AP fails for one run (the average is excluded this run).

TABLE I: Total average number of FFT calls for different methods in 10 dependent runs

\begin{tabular}{ccccc}
\hline Method & SD & NCG & LBFGS & AP \\
\hline FFT calls & 24906 & 6438 & 3963 & 17527 \\
\hline
\end{tabular}

3) Performance for Real-valued Signal: With the aid of the constraint of real-valuedness, the phase retrieval problem 


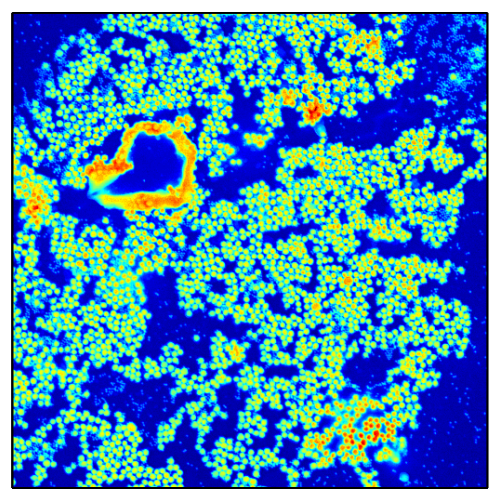

(a) Original image

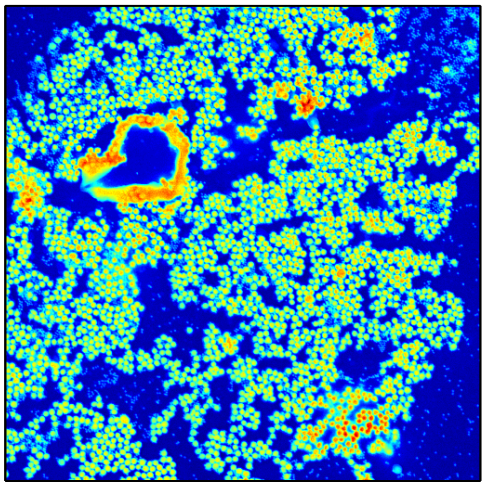

(c) Reconstruction from ten binary masks

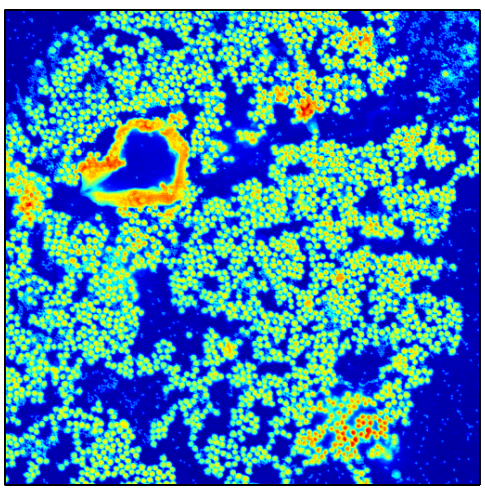

(b) Reconstruction from ten octanary masks

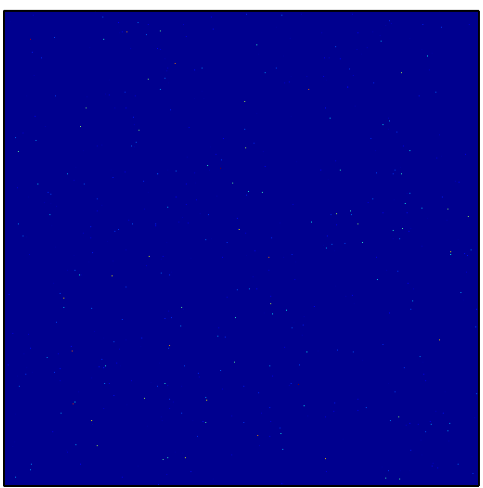

(d) Error (a) and (c)

Fig. 2: Original gold balls image and reconstructions

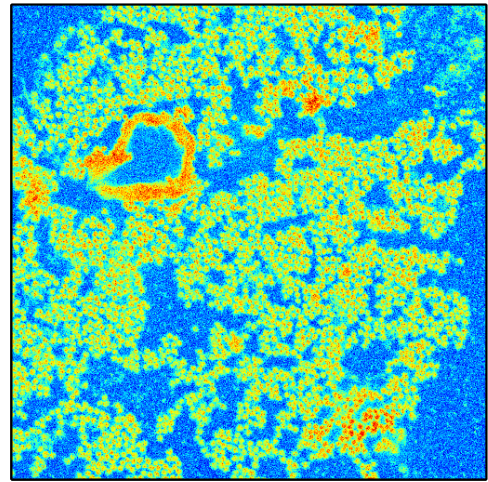

(a) Low SNR $=10 \mathrm{~dB}$, relerr $=0.2098$

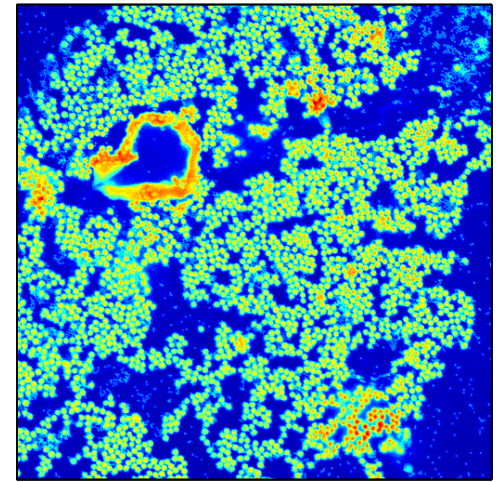

(b) Low SNR $=30 \mathrm{~dB}$, relerr $=0.0209$

Fig. 3: Reconstructions from noisy data

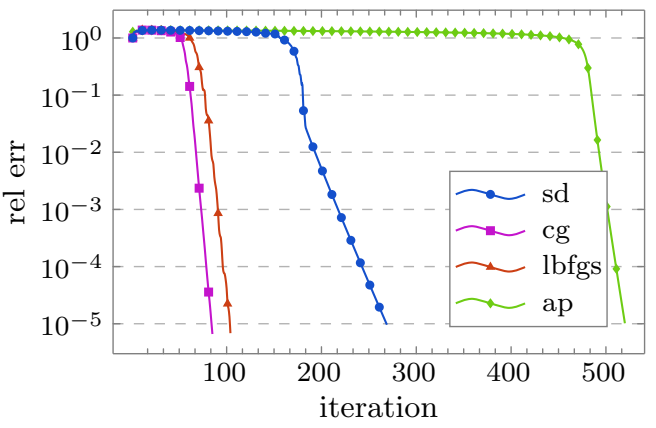

(a) Performance versus different methods

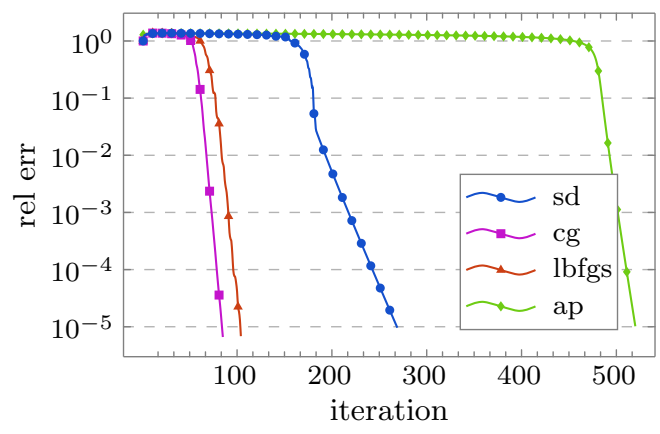

(b) Performance versus number of illuminations

Fig. 5: Comparison of different methods 


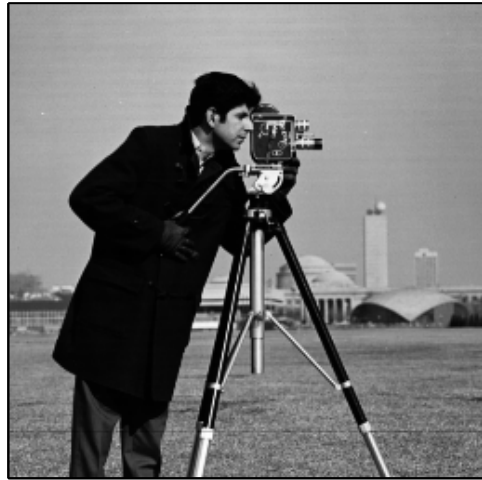

(a) Original

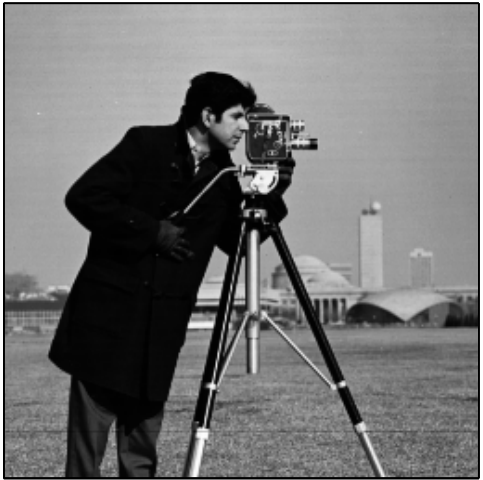

(b) Reconstruction from four masks

Fig. 6: Reconstructions of real-valued image

needs fewer diffraction patterns. Figure 6 shows the original test image and reconstructions from four octanary illuminations. Table $[\mathrm{II}$ list the iterations that algorithm returns the solution when given different number of masks. With the real assumption, the more masks are provided, the fewer iterations are needed. Even three masks can recover the solution. If without the real assumption, six illuminations are needed and the number of iterations is over 1300 .

TABLE II: Cameraman $(256 \times 256)$ image recovery with/without the real-valued information of the signal using different number of masks: the array cell is the iteration number and - stands for failure of recovery within 3000 iterations.

\begin{tabular}{ccccc}
\hline No. of masks & 3 & 4 & 5 & 6 \\
\hline real & 166 & 94 & 71 & 62 \\
complex & - & - & - & 1319 \\
\hline
\end{tabular}

\section{Conclusion And Discussion}

The generalized phase retrieval is solved by minimizing a nonconvex least squares cost function with the advantage of better practice for two dimensional problem. We deduced the expressions of gradient and Hessian of the cost function by Fréchet derivative instead of Wirtinger derivative used in literature [20]. Our approach is more convenient and clear and can be applied to other complicated cost functions. Then we design line search methods only depending gradient information for the real functions with complex variables. The advantage is that we do not need to carry out optimization algorithm with respect to real and imaginary parts of variables. We proved the global convergence of the proposed gradient decent algorithms, with arbitrary random instead of special spectral initialization, under the assumption that the sample vectors are drawn from complex Gaussian and the sampling complexity $m$ is large enough $(\mathcal{O}(n \log n)$ for Gaussian model and $\mathcal{O}\left(n \log ^{3} n\right)$ for CDP model).

As demonstrated in this paper, a priori knowledge of the real-valuedness of the signal can mitigate the phase retrieval problem. For real-valued signal, the local convexity of the least squares cost function in the neighborhood of the solution is also proved. It is may be a hinder to obtain the appropriate stepsize in the gradient descent method. For practical implementation and more rapidly convergence rate, we apply other line search methods. The study of the performance of different line search methods shows that LBFGS algorithm can efficiently and robustly solve the generalized phase retrieval problem. The nonconvexity of the cost function is not so scary is due to the premise that all local minimizers associated with the cost function are global minimizers with high probability.

Our theoretical results about global convergence are not applicable to the NCG and LBFGS algorithms, while they show more appealing performance. The convergence of NCG and BFGS for general non-convex problems is not guaranteed [27], and we cannot prove that for the generalized phase retrieval problem at present. Besides, the sampling complexity may be further reduced to $\mathcal{O}(n)$ by borrowing the idea of truncated Wirtinger flow in literate [28]. It is also worth investigating whether the performance of numerical algorithm can be further improved by exploring the structures of the signal, such as sparsity [29]. Our theoretical analysis is based on the random sampling vectors, unfortunately, the random measurements are not easy to implement, so how to reduce the randomness is the main aspect to study in phase retrieval community.

\section{ACKNOWLEDGMENTS}

The authors are indebted to Stefano Marchesini for providing us with the gold balls data set used in numerical simulations. The first author would like to thank Ms. Chao Wang for helpful comments and suggestions on this manuscript draft. This work was supported in part by NSF grants of China (61421062, 11471024). 
APPENDIX

\section{A. Lemmas and Proofs}

Lemma A.1. For matrices $A \in \mathbb{C}^{m \times n}, C \in \mathbb{C}^{m \times n}$, and $B \in$ $\mathbb{C}^{n \times m}, D \in \mathbb{C}^{n \times m}$, where $m \geq 2 n$. Then we have

$$
\begin{aligned}
& \operatorname{det}(\lambda I-A B-C D) \\
& \quad=\lambda^{m-2 n} \operatorname{det}\left(\left[\begin{array}{cc}
\lambda I-B A & B C \\
D A & \lambda I-D C
\end{array}\right]\right) .
\end{aligned}
$$

Proof: Consider matrices

$$
\widetilde{A}=\left[\begin{array}{ll}
A & C
\end{array}\right] \in \mathbb{C}^{m \times 2 n} \quad \widetilde{B}=\left[\begin{array}{l}
B \\
D
\end{array}\right] \in \mathbb{C}^{2 n \times m}
$$

According to the equality $\operatorname{det}(\lambda I-\widetilde{A} \widetilde{B})=\lambda^{m-2 n} \operatorname{det}(\lambda I-$ $\widetilde{B} \widetilde{A})$, we have

$$
\begin{array}{r}
\operatorname{det}(\lambda I-\widetilde{A} \widetilde{B})=\operatorname{det}(\lambda I-A B-C D) \\
=\lambda^{m-2 n} \operatorname{det}\left(\left[\begin{array}{cc}
\lambda I-B A & -B C \\
-D A & \lambda I-D C
\end{array}\right]\right) \\
\quad=\lambda^{m-2 n} \operatorname{det}(\lambda I-\widetilde{B} \widetilde{A}) .
\end{array}
$$

Proof of Lemma III.2. we denote $\mathbb{E}\left[\nabla^{2} f(\boldsymbol{x})\right]$ by $A$, the characteristic polynomial of the matrix $A$ is

$$
\begin{aligned}
P(\lambda)=\operatorname{det}(\lambda I-A) & \\
& =\operatorname{det}\left(\left(\lambda-\|x\|^{2}\right) I-\frac{3}{2} \boldsymbol{a} \boldsymbol{a}^{*}-\frac{1}{2} \boldsymbol{b} \boldsymbol{b}^{*}\right),
\end{aligned}
$$

where $\boldsymbol{a}=\left[\begin{array}{l}\boldsymbol{x} \\ \overline{\boldsymbol{x}}\end{array}\right] \in \mathbb{C}^{2 n}$ and $\boldsymbol{b}=i\left[\begin{array}{c}\boldsymbol{x} \\ -\overline{\boldsymbol{x}}\end{array}\right] \in \mathbb{C}^{2 n}$.

By Lemma A.1, we have

$$
\begin{aligned}
& P(\lambda)=\operatorname{det}\left(\left(\lambda-\|x\|^{2}\right) I-\frac{3}{2} \boldsymbol{a}^{*}-\frac{1}{2} \boldsymbol{b}^{*}\right) \\
= & \left(\lambda-\|\boldsymbol{x}\|^{2}\right)^{2 n-2} \operatorname{det}\left(\left(\lambda-\|\boldsymbol{x}\|^{2}\right) I-\left[\begin{array}{cc}
3\|\boldsymbol{x}\|^{2} & 0 \\
0 & -\|\boldsymbol{x}\|^{2}
\end{array}\right]\right) \\
= & \left(\lambda-\|\boldsymbol{x}\|^{2}\right)^{2 n-2} \lambda\left(\lambda-4\|\boldsymbol{x}\|^{2}\right) .
\end{aligned}
$$

So the eigenvalues are directly obtained. The proof for real case is similar.

Proof of Lemma III.4 According the expression of gradient

$$
\nabla f(\boldsymbol{z})=\frac{1}{m} \sum_{r=1}^{m}\left(\left|\boldsymbol{a}_{r}^{*} \boldsymbol{z}\right|^{2}-\left|\boldsymbol{a}_{r}^{*} \boldsymbol{x}\right|^{2}\right) \boldsymbol{a}_{r} \boldsymbol{a}_{r}^{*} \boldsymbol{z},
$$

and the expectation

$$
\mathbb{E}\left[\left|\boldsymbol{a}_{r}^{*} \boldsymbol{z}\right|^{2} \boldsymbol{a}_{r} \boldsymbol{a}_{r}^{*}\right]=\|\boldsymbol{z}\|^{2} I+\boldsymbol{z} \boldsymbol{z}^{*}, \quad \forall \boldsymbol{z} \in \mathbb{C}^{n} .
$$

So

$$
\begin{aligned}
\mathbb{E}[\nabla f(\boldsymbol{z})] & =\left(\|\boldsymbol{z}\|^{2} I+\boldsymbol{z} \boldsymbol{z}^{*}\right) \boldsymbol{z}-\left(\|\boldsymbol{x}\|^{2} I+\boldsymbol{x} \boldsymbol{x}^{*}\right) \boldsymbol{z} \\
& =\left(2\|\boldsymbol{z}\|^{2}-\|\boldsymbol{x}\|^{2}\right) \boldsymbol{z}-\left(\boldsymbol{x}^{*} \boldsymbol{z}\right) \boldsymbol{x}
\end{aligned}
$$

This completes the proof.

Proof of Lemma III.5. Let $\boldsymbol{z}=\boldsymbol{x}+$ th such that $\operatorname{Re}\left(\boldsymbol{x}^{*} \boldsymbol{h}\right)=\|\boldsymbol{x}\|,\|\boldsymbol{h}\|=1$ and $t \geq 0$. Then

$\|\nabla f(\boldsymbol{z})-\mathbb{E}[\nabla f(\boldsymbol{z})]\|=\max _{\boldsymbol{u} \in \mathbb{C}^{n},\|\boldsymbol{u}\|=1}|\langle\boldsymbol{u}, \nabla f(\boldsymbol{z})-\mathbb{E}[\nabla f(\boldsymbol{z})]\rangle|$.

By calculus, we have

$$
\begin{aligned}
\langle\boldsymbol{u}, \nabla & f(\boldsymbol{z})\rangle \\
& =\frac{1}{m} \sum_{r=1}^{m} t \boldsymbol{u}^{*}\left(\left|\boldsymbol{a}_{r}^{*} \boldsymbol{x}\right|^{2} \boldsymbol{a}_{r} \boldsymbol{a}_{r}^{*}\right) \boldsymbol{h}+t \boldsymbol{u}^{*}\left(\left(\boldsymbol{a}_{r}^{*} \boldsymbol{x}\right)^{2} \boldsymbol{a}_{r} \boldsymbol{a}_{r}^{T}\right) \overline{\boldsymbol{h}} \\
& +2 t^{2} \boldsymbol{u}^{*}\left(\left|\boldsymbol{a}_{r}^{*} \boldsymbol{h}\right|^{2} \boldsymbol{a}_{r} \boldsymbol{a}_{r}^{*}\right) \boldsymbol{x}+t^{2} \boldsymbol{u}^{*}\left(\left(\boldsymbol{a}_{r}^{*} \boldsymbol{h}\right)^{2} \boldsymbol{a}_{r} \boldsymbol{a}_{r}^{T}\right) \overline{\boldsymbol{x}} \\
& +t^{3} \boldsymbol{u}^{*}\left(\left|\boldsymbol{a}_{r}^{*} \boldsymbol{h}\right|^{2} \boldsymbol{a}_{r} \boldsymbol{a}_{r}^{*}\right) \boldsymbol{h} .
\end{aligned}
$$

By Lemma III.4, we have

$$
\begin{aligned}
\mathbb{E}[\nabla f(\boldsymbol{z})]=2 t\left(t^{2}+3 t\|\boldsymbol{x}\|\right. & \left.+2\|\boldsymbol{x}\|^{2}\right) \boldsymbol{h}, \text { and } \\
\|\mathbb{E}[\nabla f(\boldsymbol{z})]\| & =2 t\left(t^{2}+3 t\|\boldsymbol{x}\|+2\|\boldsymbol{x}\|^{2}\right) .
\end{aligned}
$$

Furthermore, we also have

$$
\begin{aligned}
\langle\boldsymbol{u}, \mathbb{E}[\nabla & f(\boldsymbol{z})]\rangle \\
& =t \boldsymbol{u}^{*}\left(\|\boldsymbol{x}\|^{2} I+\boldsymbol{x} \boldsymbol{x}^{*}\right) \boldsymbol{h}+t \boldsymbol{u}^{*}\left(2 \boldsymbol{x} \boldsymbol{x}^{T}\right) \overline{\boldsymbol{h}} \\
& +2 t^{2} \boldsymbol{u}^{*}\left(\|\boldsymbol{h}\|^{2} I+\boldsymbol{h} \boldsymbol{h}^{*}\right) \boldsymbol{x}+t^{2} \boldsymbol{u}^{*}\left(2 \boldsymbol{h} \boldsymbol{h}^{T}\right) \overline{\boldsymbol{x}} \\
& +t^{3} \boldsymbol{u}^{*}\left(\|\boldsymbol{h}\|^{2} I+\boldsymbol{h} \boldsymbol{h}^{*}\right) \boldsymbol{h}
\end{aligned}
$$

where we use the equality $2\|\boldsymbol{h}\|^{2} \boldsymbol{u}^{*} \boldsymbol{h}=$ $\boldsymbol{u}^{*}\left(\|\boldsymbol{h}\|^{2} I+\boldsymbol{h} \boldsymbol{h}^{*}\right) \boldsymbol{h}$.

Combining the two equalities 23 and (26) together and using triangular inequality and Lemma III.3 give

$$
\begin{aligned}
|\langle\boldsymbol{u}, \nabla f(\boldsymbol{z})-\mathbb{E}[\nabla f(\boldsymbol{z})]\rangle| & \\
\leq & t\left|\boldsymbol{u}^{*}\left(\frac{1}{m} \sum_{r=1}^{m}\left|\boldsymbol{a}_{r}^{*} \boldsymbol{x}\right|^{2} \boldsymbol{a}_{r} \boldsymbol{a}_{r}^{*}-\left(\|\boldsymbol{x}\|^{2} I+\boldsymbol{x} \boldsymbol{x}^{*}\right)\right) \boldsymbol{h}\right| \\
& +t\left|\boldsymbol{u}^{*}\left(\frac{1}{m} \sum_{r=1}^{m}\left(\boldsymbol{a}_{r}^{*} \boldsymbol{x}\right)^{2} \boldsymbol{a}_{r} \boldsymbol{a}_{r}^{T}-2 \boldsymbol{x} \boldsymbol{x}^{T}\right) \overline{\boldsymbol{h}}\right| \\
& +2 t^{2}\left|\boldsymbol{u}^{*}\left(\frac{1}{m} \sum_{r=1}^{m}\left|\boldsymbol{a}_{r}^{*} \boldsymbol{h}\right|^{2} \boldsymbol{a}_{r} \boldsymbol{a}_{r}^{*}-\left(\|\boldsymbol{h}\|^{2} I+\boldsymbol{h} \boldsymbol{h}^{*}\right)\right) \boldsymbol{x}\right| \\
& +t^{2}\left|\boldsymbol{u}^{*}\left(\frac{1}{m} \sum_{r=1}^{m}\left(\boldsymbol{a}_{r}^{*} \boldsymbol{h}\right)^{2} \boldsymbol{a}_{r} \boldsymbol{a}_{r}^{T}-2 \boldsymbol{h} \boldsymbol{h}^{T}\right) \overline{\boldsymbol{x}}\right| \\
& +t^{3}\left|\boldsymbol{u}^{*}\left(\frac{1}{m} \sum_{r=1}^{m}\left|\boldsymbol{a}_{r}^{*} \boldsymbol{h}\right|^{2} \boldsymbol{a}_{r} \boldsymbol{a}_{r}^{*}-\left(\|\boldsymbol{h}\|^{2} I+\boldsymbol{h} \boldsymbol{h}^{*}\right)\right) \boldsymbol{h}\right| \\
\leq & 2 t \delta\|\boldsymbol{x}\|^{2}+3 t^{2} \delta\|\boldsymbol{x}\|+t^{3} \delta \\
= & \frac{\delta}{2}\|\mathbb{E}[\nabla f(\boldsymbol{z})]\| .
\end{aligned}
$$

Take $t=\operatorname{dist}(\boldsymbol{z}, \boldsymbol{x}) \leq\|\boldsymbol{x}\| / 2$, then

$$
t^{2}+3 t\|\boldsymbol{x}\|+2\|\boldsymbol{x}\|^{2} \leq \frac{15}{4}\|\boldsymbol{x}\|^{2} \leq 4\|\boldsymbol{x}\|^{2} .
$$


So

$$
|\langle\boldsymbol{u}, \nabla f(\boldsymbol{z})-\mathbb{E}[\nabla f(\boldsymbol{z})]\rangle| \leq 4 \delta \operatorname{dist}(\boldsymbol{z}, \boldsymbol{x})\|\boldsymbol{x}\|^{2} .
$$

Proof of Lemma III.6. Since we have

$$
\mathbb{E}[\nabla f(\boldsymbol{z})]=2 t\left(t^{2}+3 t\|\boldsymbol{x}\|+2\|\boldsymbol{x}\|^{2}\right) \boldsymbol{h},
$$

it is obvious that $\mathbb{E}[\nabla f(\boldsymbol{z})]$ and $\boldsymbol{h}$ share the same direction. Furthermore, we have

$$
\begin{aligned}
\|\mathbb{E}[\nabla f(\boldsymbol{z})]\|=2 t & \left(t^{2}+3 t\|\boldsymbol{x}\|+2\|\boldsymbol{x}\|^{2}\right) \\
& =2 t\left(\left(t+\frac{3}{2}\|\boldsymbol{x}\|\right)^{2}-\frac{1}{4}\|\boldsymbol{x}\|^{2}\right) \geq 0,
\end{aligned}
$$

so $\mathbb{E}[\nabla f(\boldsymbol{z})]=\mathbf{0}$ if and only if $\boldsymbol{z}$ is a solution of the phase retrieval problem (1). Furthermore, by equation (15), it follows that

$\operatorname{Re}\left\langle\nabla f(\boldsymbol{z}), \boldsymbol{z}-\boldsymbol{x} e^{i \phi(\boldsymbol{z})}\right\rangle \geq \sqrt{1-\frac{\delta^{2}}{4}}\|\nabla f(\boldsymbol{z})\|\left\|\boldsymbol{z}-\boldsymbol{x} e^{i \phi(\boldsymbol{z})}\right\|$ so the angle between $\nabla f(\boldsymbol{z})$ and $\boldsymbol{z}-\boldsymbol{x} e^{i \phi(\boldsymbol{z})}$ is below $\arcsin (\delta / 2)$.

\section{B. Proof of Theorem III.7}

Proof: According to LemmaIII.6, the angle between $z_{k}-$ $\boldsymbol{x} e^{i \phi\left(\boldsymbol{z}_{k}\right)}$ and $\nabla f\left(\boldsymbol{z}_{k}\right)$ is below $\pi / 2$. It is obvious that if $0<$ $\alpha_{k}<2 \operatorname{Re}\left\langle\boldsymbol{z}_{k}-\boldsymbol{x} e^{i \phi\left(\boldsymbol{z}_{k}\right)}, \nabla f\left(\boldsymbol{z}_{k}\right)\right\rangle /\left\|\nabla f\left(\boldsymbol{z}_{k}\right)\right\|$, according to the definition of $\phi\left(\boldsymbol{z}_{k+1}\right)$, we have

$\left\|\boldsymbol{z}_{k+1}-\boldsymbol{x} e^{i \phi\left(\boldsymbol{z}_{k+1}\right)}\right\| \leq\left\|\boldsymbol{z}_{k+1}-\boldsymbol{x} e^{i \phi\left(\boldsymbol{z}_{k}\right)}\right\|<\left\|\boldsymbol{z}_{k}-\boldsymbol{x} e^{i \phi\left(\boldsymbol{z}_{k}\right)}\right\|$, i.e., the strict descent property $\operatorname{dist}\left(\boldsymbol{z}_{k+1}, \boldsymbol{x}\right)<\operatorname{dist}\left(\boldsymbol{z}_{k}, \boldsymbol{x}\right)$ holds.

So we have to bound the $\left\|\boldsymbol{z}_{k}-\boldsymbol{x} e^{i \phi\left(\boldsymbol{z}_{k}\right)}\right\|$. For brevity, we omit the subscript $k$ without ambiguity and assume the $\phi(\boldsymbol{z})=0$. Let $\boldsymbol{z}=\boldsymbol{x}+t \boldsymbol{h}$ such that $\operatorname{Re}\left(\boldsymbol{x}^{*} \boldsymbol{h}\right)=\|\boldsymbol{x}\|,\|\boldsymbol{h}\|=$ 1 and $t \geq 0$, so $\operatorname{dist}(\boldsymbol{z}, \boldsymbol{x})=t$. According to (15) in Lemma III.5, we have

$\|\nabla f(\boldsymbol{z})\| \leq\left(1+\frac{\delta}{2}\right)\|\mathbb{E}[\nabla f(\boldsymbol{z})]\| \leq(2+\delta) t\left(t^{2}+3 t\|\boldsymbol{x}\|+2\|\boldsymbol{x}\|^{2}\right)$.

We have

$$
\|\nabla f(\boldsymbol{z})\| \leq \begin{cases}6(2+\delta)\|\boldsymbol{x}\|^{2} t, & t \leq\|\boldsymbol{x}\| \\ 6(2+\delta) t^{3}, & t>\|\boldsymbol{x}\| .\end{cases}
$$

Consequently, we have

$$
t \geq \begin{cases}\frac{\|\nabla f(\boldsymbol{z})\|}{6(2+\delta)\|\boldsymbol{x}\|^{2}}, & t \leq\|\boldsymbol{x}\| \\ \sqrt[3]{\frac{\|\nabla f(\boldsymbol{z})\|}{6(2+\delta)}}, & t>\|\boldsymbol{x}\|\end{cases}
$$

Thus, according to Lemma III.6, we have

$$
\begin{aligned}
2 \operatorname{Re}\left\langle\boldsymbol{z}-\boldsymbol{x} e^{i \phi(\boldsymbol{z})}, \nabla f(\boldsymbol{z})\right\rangle / & \|\nabla f(\boldsymbol{z})\| \\
& \geq 2 \sqrt{1-\frac{\delta^{2}}{4}}\left\|\boldsymbol{z}-\boldsymbol{x} e^{i \phi(\boldsymbol{z})}\right\| .
\end{aligned}
$$

It follows that

$$
\begin{aligned}
& 0<\alpha_{k} \\
& <\min \left\{2 \sqrt{1-\frac{\delta^{2}}{4}} \frac{\left\|\nabla f\left(\boldsymbol{z}_{k}\right)\right\|}{6(2+\delta)\|\boldsymbol{x}\|^{2}}, 2 \sqrt{1-\frac{\delta^{2}}{4}} \sqrt[3]{\frac{\left\|\nabla f\left(\boldsymbol{z}_{k}\right)\right\|}{6(2+\delta)}}\right\} .
\end{aligned}
$$

For the proof of convergence, the stepsize $\alpha_{k}$ can not be too small. We denote the intersection of the polynomial of degree three $P_{2}(t)=(2-\delta) t\left(t^{2}+3 t\|\boldsymbol{x}\|+2\|\boldsymbol{x}\|^{2}\right)$ and $y=$ $\left\|\nabla f\left(\boldsymbol{z}_{k}\right)\right\|$ as $t_{2}$. We choose $\alpha_{k}=\sqrt{1-\delta^{2} / 4} t_{1}$. The proof of convergence is followed. According to (15) in Lemma III.5. since we have the following inequality

$$
\left(1-\frac{\delta}{2}\right)\left\|\mathbb{E}\left[\nabla f\left(\boldsymbol{z}_{k}\right)\right]\right\| \leq\left\|\nabla f\left(\boldsymbol{z}_{k}\right)\right\| \leq\left(1+\frac{\delta}{2}\right)\left\|\mathbb{E}\left[\nabla f\left(\boldsymbol{z}_{k}\right)\right]\right\|,
$$

where the quality

$$
\left\|\mathbb{E}\left[\nabla f\left(\boldsymbol{z}_{k}\right)\right]\right\|=2 t\left(t^{2}+3 t\|\boldsymbol{x}\|+2\|x\|^{2}\right) .
$$

It is obvious that from (27) we can bound the variable $t$ for a given $\left\|\nabla f\left(\boldsymbol{z}_{k}\right)\right\|$.

From Figure 7, we have that

$$
\frac{|S T|}{|O T|} \leq \frac{|S T|}{\left|O T_{1}\right|} .
$$

We take $S=\left(t_{1} \cos \beta, 0\right)$ and $T=(t \cos \beta, t \sin \beta)$, where $t \in\left[t_{1}, t_{2}\right]$. It follows that

$$
\begin{aligned}
\frac{|S T|^{2}}{\left|O T_{1}\right|^{2}} & =\frac{\left(t-t_{1}\right)^{2} \cos ^{2} \beta+t^{2} \sin ^{2} \beta}{t_{1}^{2}} \\
& =\left(\frac{t-t_{1}}{t_{1}}\right)^{2} \cos ^{2} \beta+\left(1+\frac{t-t_{1}}{t_{1}}\right)^{2} \sin ^{2} \beta \\
& \leq\left(\frac{t-t_{1}}{t_{1}}\right)^{2} \cos ^{2} \beta+2 \sin ^{2} \beta+2\left(\frac{t-t_{1}}{t_{1}}\right)^{2} \sin ^{2} \beta \\
& \leq 2\left(\frac{t-t_{1}}{t_{1}}\right)^{2}+2 \sin ^{2} \beta
\end{aligned}
$$

Since the $P_{1}$ and $P_{2}$ are both convex when $t \geq 0$, we have the inequality

$$
\frac{t-t_{1}}{t_{1}} \leq \frac{2 \delta\left(t_{1}^{2}+3 t_{1}+2\right)}{(2-\delta)\left(3 t_{1}^{2}+6 t_{1}+2\right)}:=f\left(t_{1}\right),
$$

where we assume $\|\boldsymbol{x}\|=1$ without loss of generality. It is obvious that $f\left(t_{1}\right)$ monotonously decreases first and then monotonously increases. As $f\left(t_{1}\right) \rightarrow 2 \delta /(3(2-\delta)), t_{1} \rightarrow \infty$, we have

$$
\begin{aligned}
\frac{|S T|^{2}}{\left|O T_{1}\right|^{2}} \leq 2 & \left(\frac{2 \delta}{2-\delta}\right)^{2}+2 \sin ^{2} \beta \\
& \leq 2\left(\frac{2 \delta}{2-\delta}\right)^{2}+\frac{\delta^{2}}{2} \leq\left(\frac{2 \sqrt{2} \delta}{2-\delta}+\frac{\delta}{\sqrt{2}}\right)^{2}
\end{aligned}
$$

That is

$$
|S T| \leq\left(\frac{2 \sqrt{2} \delta}{2-\delta}+\frac{\delta}{\sqrt{2}}\right)\left|O T_{1}\right| \leq\left(\frac{2 \sqrt{2} \delta}{2-\delta}+\frac{\delta}{\sqrt{2}}\right)|O T|,
$$




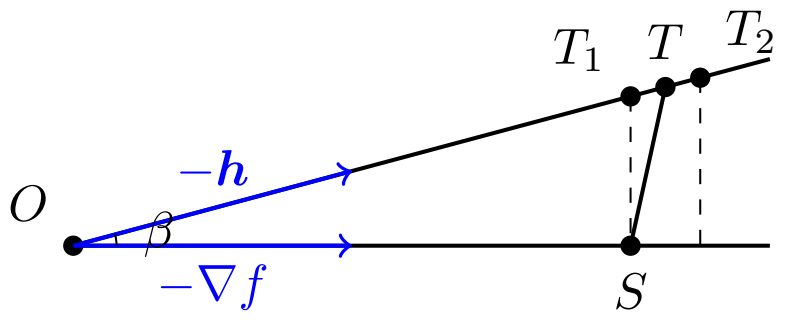

Fig. 7: Illustration of the descent property: point $T$ is between $T_{1}$ and $T_{2}$, and $\left|O T_{1}\right|=t_{1}$ and $\left|O T_{2}\right|=t_{2}$, the step size $|O S|=t_{1} \sin \beta$, where $\beta$ is the angle between $-\boldsymbol{h}$ and $-\nabla f$.

i.e., the convergence is ensured. It yields that

$$
\operatorname{dist}\left(\boldsymbol{z}_{k+1}, \boldsymbol{x}\right) \leq\left(\frac{2 \sqrt{2} \delta}{2-\delta}+\frac{\delta}{\sqrt{2}}\right) \operatorname{dist}\left(\boldsymbol{z}_{k}, \boldsymbol{x}\right) .
$$

Since the $g(\delta)=\frac{6 \delta-\delta^{2}}{\sqrt{2}(2-\delta)}$ monotonously increases, we can find small enough $\delta$ to satisfy the condition $g(\delta)<1$, such as $\delta \leq 0.2$, then $g(\delta)<0.5$.

Proof of Corollary III.8. If we have $t \leq \frac{1}{5}\|\boldsymbol{x}\|$, then it follows that

$\left\|\nabla f\left(\boldsymbol{z}_{k}\right)\right\| \leq(2+\delta) t\left(t^{2}+3 t\|\boldsymbol{x}\|+2\|\boldsymbol{x}\|^{2}\right) \leq(2+\delta) \frac{66}{25}\|\boldsymbol{x}\|^{2} t$.

Then we have

$$
t \geq \frac{25\left\|\nabla f\left(\boldsymbol{z}_{k}\right)\right\|}{66(2+\delta)\|\boldsymbol{x}\|^{2}}
$$

We have the estimations

$\frac{t-t_{1}}{t_{1}} \leq \frac{66(2+\delta) / 25-(2-\delta)\left(t_{1}^{2}+3 t_{1}+2\right)}{(2-\delta)\left(3 t_{1}^{2}+6 t_{1}+2\right)} \leq \frac{58 \delta+16}{25(2-\delta)}$,

and

$$
\frac{|S T|^{2}}{\left|O T_{1}\right|^{2}} \leq 2\left(\frac{58 \delta+16}{25(2-\delta)}\right)^{2}+\frac{\delta^{2}}{2} \leq\left(\frac{\sqrt{2}(58 \delta+16)}{25(2-\delta)}+\frac{\delta}{\sqrt{2}}\right)^{2} .
$$

For the case $\operatorname{dist}\left(\boldsymbol{z}_{k}, \boldsymbol{x}\right) \geq 2\|\boldsymbol{x}\|$, the proof is similar, we omit it.

\section{Proof of Local Convexity}

Proof of Theorem III.11. Taking an arbitrary $\boldsymbol{z} \in \mathbb{R}^{n}$, we let $\boldsymbol{z}=\boldsymbol{x}+t \boldsymbol{w}(\|w\|=1, t \geq 0)$. According to Lemma III.9. it yields

$$
\begin{aligned}
& \mathbb{E}\left[\nabla^{2} f(\boldsymbol{z})\right] \\
& \quad=\mathbb{E}\left[\nabla^{2} f(\boldsymbol{x}+t \boldsymbol{w})\right]=\left(I+4 \boldsymbol{w} \boldsymbol{w}^{T}\right) t^{2} \\
& +4\left(\left(\boldsymbol{x}^{T} \boldsymbol{w}\right) I+\boldsymbol{x} \boldsymbol{w}^{T}+\boldsymbol{w} \boldsymbol{x}^{T}\right) t+\left(\|\boldsymbol{x}\|^{2} I+3 \boldsymbol{x} \boldsymbol{x}^{T}\right) .
\end{aligned}
$$

Observe that

$$
\left(\boldsymbol{x}^{T} \boldsymbol{w}\right) I+\boldsymbol{x} \boldsymbol{w}^{T}+\boldsymbol{w} \boldsymbol{x}^{T} \succeq-3\|\boldsymbol{x}\| I
$$

and

$$
\|\boldsymbol{x}\|^{2} I+3 \boldsymbol{x} \boldsymbol{x}^{T} \succeq\|\boldsymbol{x}\|^{2} I .
$$

So, when $t \leq\|\boldsymbol{x}\| / 12$, the expectation $\mathbb{E}\left[\nabla^{2} f(\boldsymbol{z})\right] \succeq 0$. This completes the proof.

Lemma A.2. For a given vector $\boldsymbol{z} \in \mathbb{R}^{n}$, we parameterize it by $\boldsymbol{z}=\boldsymbol{x}+t \boldsymbol{w}$, where $\boldsymbol{w}=1, t \geq 0$. Then according to the chain rule of Wirtinger derivatives, we have

$$
f(\boldsymbol{z})=f(\boldsymbol{x}+t \boldsymbol{w})=\frac{1}{2 m}\left\||A(\boldsymbol{x}+t \boldsymbol{w})|^{2}-\boldsymbol{y}\right\|^{2} .
$$

Its gradient and Hessian with respect to $t$ are:

$f^{\prime}(t)$

$$
\begin{aligned}
& =\frac{1}{m} \sum_{r=1}^{m}\left[\begin{array}{ll}
\boldsymbol{w}^{T} & \boldsymbol{w}^{*}
\end{array}\right]\left[\begin{array}{l}
\left(\left|\boldsymbol{a}_{r}^{*}(\boldsymbol{x}+t \boldsymbol{w})\right|^{2}-y_{r}\right) \overline{\left(\boldsymbol{a}_{r}^{*}(\boldsymbol{x}+t \boldsymbol{w})\right.} \overline{\boldsymbol{a}_{r}} \\
\left(\left|\boldsymbol{a}_{r}^{*}(\boldsymbol{x}+t \boldsymbol{w})\right|^{2}-y_{r}\right)\left(\boldsymbol{a}_{r}^{*}(\boldsymbol{x}+t \boldsymbol{w})\right) \boldsymbol{a}_{r}
\end{array}\right] \\
& =\frac{2}{m} \sum_{r=1}^{m} \operatorname{Re}\left(\left(\left|\boldsymbol{a}_{r}^{*}(\boldsymbol{x}+t \boldsymbol{w})\right|^{2}-y_{r}\right) \boldsymbol{a}_{r}^{*}(\boldsymbol{x}+t \boldsymbol{w}) \boldsymbol{w}^{*} \boldsymbol{a}_{r}\right) \text {, } \\
& f^{\prime \prime}(t)=\frac{1}{m} \sum_{r=1}^{m}\left[\begin{array}{ll}
\boldsymbol{w}^{*} & \boldsymbol{w}^{T}
\end{array}\right] \\
& {\left[\begin{array}{cc}
\left(2\left|\boldsymbol{a}_{r}^{*}(\boldsymbol{x}+t \boldsymbol{w})\right|^{2}-y_{r}\right) \boldsymbol{a}_{r} \boldsymbol{a}_{r}^{*} & \left(\boldsymbol{a}_{r}^{*}(\boldsymbol{x}+t \boldsymbol{w})\right)^{2} \boldsymbol{a}_{r} \boldsymbol{a}_{r}^{T} \\
\left(\overline{\boldsymbol{a}_{r}^{*}(\boldsymbol{x}+t \boldsymbol{w})}\right)^{2} \overline{\boldsymbol{a}_{r}} \boldsymbol{a}_{r}^{*} & \left(2\left|\boldsymbol{a}_{r}^{*}(\boldsymbol{x}+t \boldsymbol{w})\right|^{2}-y_{r}\right) \overline{\boldsymbol{a}_{r}} \boldsymbol{a}_{r}^{T}
\end{array}\right]\left[\begin{array}{l}
\boldsymbol{w} \\
\overline{\boldsymbol{w}}
\end{array}\right]} \\
& =\frac{2}{m} \sum_{r=1}^{m} \operatorname{Re}\left(\left(2\left|\boldsymbol{a}_{r}^{*}(\boldsymbol{x}+t \boldsymbol{w})\right|^{2}-y_{r}\right)\left|\boldsymbol{a}_{r}^{*} \boldsymbol{w}\right|^{2}\right. \\
& \left.+\left(\boldsymbol{a}_{r}^{*}(\boldsymbol{x}+t \boldsymbol{w})\right)^{2}\left(\boldsymbol{a}_{r}^{T} \overline{\boldsymbol{w}}\right)^{2}\right) .
\end{aligned}
$$

Proof of Theorem III.12. By Lemma A.2, we have

$$
\begin{aligned}
& \frac{1}{2}\left[\begin{array}{ll}
\boldsymbol{w}^{*} & \boldsymbol{w}^{T}
\end{array}\right] \nabla^{2} f(\boldsymbol{z})\left[\begin{array}{l}
\boldsymbol{w} \\
\overline{\boldsymbol{w}}
\end{array}\right]=\frac{1}{2} f^{\prime \prime}(t) \\
& =\frac{1}{m} \sum_{r=1}^{m} \operatorname{Re}\left(3\left|\boldsymbol{a}_{r}^{*} \boldsymbol{w}\right|^{4} t^{2}+4 \operatorname{Re}\left(\boldsymbol{w}^{*} \boldsymbol{a}_{r} \boldsymbol{a}_{r}^{*} \boldsymbol{x}\right)\left|\boldsymbol{a}_{r}^{*} \boldsymbol{w}\right|^{2} t\right. \\
& \left.+2\left(\boldsymbol{w}^{*} \boldsymbol{a}_{r} \boldsymbol{a}_{r}^{*} \boldsymbol{x}\right)\left|\boldsymbol{a}_{r}^{*} \boldsymbol{w}\right|^{2} t\right)+\frac{1}{2} f^{\prime \prime}(0) \\
& =\frac{1}{m} \sum_{r=1}^{m} 3\left|\boldsymbol{a}_{r}^{*} \boldsymbol{w}\right|^{4} t^{2}+6 \operatorname{Re}\left(\boldsymbol{w}^{*} \boldsymbol{a}_{r} \boldsymbol{a}_{r}^{*} \boldsymbol{x}\right)\left|\boldsymbol{a}_{r}^{*} \boldsymbol{w}\right|^{2} t \\
& +\left|\boldsymbol{a}_{r}^{*} \boldsymbol{x}\right|^{2}\left|\boldsymbol{a}_{r}^{*} \boldsymbol{w}\right|^{2}+\operatorname{Re}\left(\left(\boldsymbol{w}^{*} \boldsymbol{a}_{r} \boldsymbol{a}_{r}^{*} \boldsymbol{x}\right)^{2}\right) \\
& =\frac{1}{m} \sum_{r=1}^{m} 3\left|\boldsymbol{a}_{r}^{*} \boldsymbol{w}\right|^{4} t^{2}+6 \operatorname{Re}\left(\boldsymbol{w}^{*} \boldsymbol{a}_{r} \boldsymbol{a}_{r}^{*} \boldsymbol{x}\right)\left|\boldsymbol{a}_{r}^{*} \boldsymbol{w}\right|^{2} t \\
& +2\left(\operatorname{Re}\left(\boldsymbol{w}^{*} \boldsymbol{a}_{r} \boldsymbol{a}_{r}^{*} \boldsymbol{x}\right)\right)^{2},
\end{aligned}
$$

where we use the equality $2 \operatorname{Re}(c)^{2}=|c|^{2}+\operatorname{Re}\left(c^{2}\right)$.

We can denote it

$$
\begin{aligned}
\frac{1}{2} & {\left[\begin{array}{ll}
\boldsymbol{w}^{*} & \boldsymbol{w}^{T}
\end{array}\right] \nabla^{2} f(\boldsymbol{z})\left[\begin{array}{l}
\boldsymbol{w} \\
\boldsymbol{w}
\end{array}\right] } \\
& =\frac{3}{m} \sum_{r=1}^{m}\left(A_{r} t+B_{r}\right)^{2}-\frac{1}{4}\left[\begin{array}{ll}
\boldsymbol{w}^{*} & \boldsymbol{w}^{T}
\end{array}\right] \nabla^{2} f(\boldsymbol{x})\left[\begin{array}{l}
\boldsymbol{w} \\
\overline{\boldsymbol{w}}
\end{array}\right],
\end{aligned}
$$

where

$$
\begin{aligned}
& A_{r}=\left|\boldsymbol{a}_{r}^{*} \boldsymbol{w}\right|^{2} \\
& B_{r}=\operatorname{Re}\left(\boldsymbol{w}^{*} \boldsymbol{a}_{r} \boldsymbol{a}_{r}^{*} \boldsymbol{x}\right) .
\end{aligned}
$$


Define

$$
Z_{r}(t)=\left(A_{r} t+B_{r}\right)^{2} \geq 0
$$

according to Lemma III.10, we have

$$
\begin{aligned}
& \mathbb{E}\left[Z_{r}(t)\right]=\mathbb{E}\left[\left|\boldsymbol{a}_{r}^{*} \boldsymbol{w}\right|^{4} t^{2}+2 \operatorname{Re}\left(\boldsymbol{w}^{*} \boldsymbol{a}_{r} \boldsymbol{a}_{r}^{*} \boldsymbol{x}\right)\left|\boldsymbol{a}_{r}^{*} \boldsymbol{w}\right|^{2} t\right. \\
& \left.+\left(\operatorname{Re}\left(\boldsymbol{w}^{*} \boldsymbol{a}_{r} \boldsymbol{a}_{r}^{*} \boldsymbol{x}\right)\right)^{2}\right]
\end{aligned}
$$

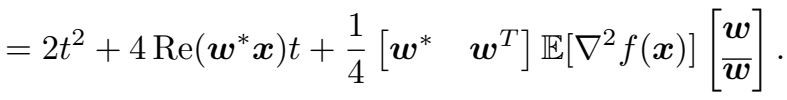

In the real case, we have $\boldsymbol{w}^{*}=\boldsymbol{w}^{T}$ and

$$
\begin{aligned}
\boldsymbol{w}^{T} \nabla^{2} f(\boldsymbol{z}) \boldsymbol{w} & =\frac{1}{2} f^{\prime \prime}(t) \\
& =\frac{3}{m} \sum_{r=1}^{m} Z_{r}(t)-\frac{1}{2} \boldsymbol{w}^{T} \nabla^{2} f(\boldsymbol{x}) \boldsymbol{w} .
\end{aligned}
$$

Consequently, it follows that the expectation

$$
\mathbb{E}\left[Z_{r}(t)\right]=2 t^{2}+4 \boldsymbol{w}^{T} \boldsymbol{x} t+\frac{1}{2} \boldsymbol{w}^{T} \mathbb{E}\left[\nabla^{2} f(\boldsymbol{x})\right] \boldsymbol{w}
$$

and that the variance

$$
\mathbb{E}\left[\left(Z_{r}(t)-\mathbb{E}\left[Z_{r}(t)\right]\right)^{2}\right] \leq C^{2}(t)
$$

where $C(t)$ depends only on the $\boldsymbol{a}_{r}$.

Applying Lemma 5.4 in literature [30] yields (taking $y=$ $m \lambda / 12)$

$$
\begin{aligned}
& \mathbb{P}\left[\mathbb{E}\left[Z_{r}(t)\right]-\frac{1}{m} \sum_{r=1}^{m} Z_{r}(t) \geq \frac{\lambda}{12}\right] \\
& \leq \min \left\{\exp \left(-\frac{\lambda^{2} m}{144 C^{2}(t)}\right), 25\left(1-\Phi\left(\frac{\lambda \sqrt{m}}{12 C(t)}\right)\right)\right\} .
\end{aligned}
$$

Using the well-known bound

$$
1-\Phi\left(\frac{\lambda \sqrt{m}}{12 C(t)}\right)<\frac{12 C(t)}{\lambda \sqrt{2 m \pi}} \exp \left(-\frac{\lambda^{2} m}{288 C^{2}(t)}\right),
$$

we find that if $m \geq 288 \alpha \lambda^{-2} C^{2}(t) n$ then with probability at least $1-e^{-\alpha n}$ we have

$$
\begin{aligned}
\frac{1}{2} f^{\prime \prime}(t) & \geq 3 \mathbb{E}\left[Z_{r}(t)\right]-\lambda / 4-\frac{1}{2} \boldsymbol{w}^{T} \nabla^{2} f(\boldsymbol{x}) \boldsymbol{w} \\
& \geq 6 t^{2}+12 \boldsymbol{w}^{T} \boldsymbol{x} t+\boldsymbol{w}^{T} \mathbb{E}\left[\nabla^{2} f(\boldsymbol{x})\right] \boldsymbol{w}-\lambda / 4 \\
& +\frac{1}{2} \boldsymbol{w}^{T} \mathbb{E}\left[\nabla^{2} f(\boldsymbol{x})\right] \boldsymbol{w}-\frac{1}{2} \boldsymbol{w}^{T} \nabla^{2} f(\boldsymbol{x}) \boldsymbol{w} \\
& \geq 6 t^{2}+12 \boldsymbol{w}^{T} \boldsymbol{x} t+\lambda / 2,
\end{aligned}
$$

where we use the concentration of the Hessian around its mean from Lemma II.3.

Since the smallest eigenvalue of $\mathbb{E}\left[\nabla^{2} f(\boldsymbol{x})\right]$ is $\|\boldsymbol{x}\|^{2}$ (see Lemma III.2, taking $\lambda=\|\boldsymbol{x}\|^{2}$, it follows that

$$
\frac{1}{2} f^{\prime \prime}(t) \geq 6 t^{2}+12 \boldsymbol{w}^{T} \boldsymbol{x} t+\|\boldsymbol{x}\|^{2} / 2 .
$$

So when $0 \leq t \leq\|\boldsymbol{x}\| / 24, f^{\prime \prime}(t) \geq 0$ holds with high probability.

\section{REFERENCES}

[1] R. P. Millane, "Phase retrieval in crystallography and optics," vol. 7, no. 3, pp. 394-411, 1990. [Online]. Available: http: //dx.doi.org/10.1364/josaa.7.000394

[2] D. L. Misell, "A method for the solution of the phase problem in electron microscopy," vol. 6, no. 1, p. L6, jan 1973. [Online]. Available: http://dx.doi.org/10.1088/0022-3727/6/1/102

[3] Y. Shechtman, Y. C. Eldar, O. Cohen, H. N. Chapman, J. Miao, and M. Segev, "Phase retrieval with application to optical imaging: A contemporary overview," vol. 32, no. 3, pp. 87-109, may 2015. [Online]. Available: http://dx.doi.org/10.1109/msp.2014.2352673

[4] T. I. Kuznetsova, "On the phase retrieval problem in optics," vol. 31, no. 4, p. 364, apr 1988. [Online]. Available: http: //dx.doi.org/10.1070/pu1988v031n04abeh005755

[5] J. R. Fienup, "Phase retrieval algorithms: A comparison," vol. 21, no. 15 , pp. 2758-2769, 1982. [Online]. Available: http://dx.doi.org/10. $1364 /$ ao. 21.002758

[6] M. H. Hayes, "The reconstruction of a multidimensional sequence from the phase or magnitude of its fourier transform," vol. 30, no. 2, pp. 140-154, apr 1982. [Online]. Available: http://dx.doi.org/10.1109/tassp. 1982.1163863

[7] M. H. Hayes and J. H. Mcclellan, "Reducible polynomials in more than one variable," vol. 70, no. 2, pp. 197-198, 1982. [Online]. Available: http://dx.doi.org/10.1109/proc.1982.12262

[8] D. R. Luke, H. H. Bauschke, and P. L. Combettes, "Hybrid projection-reflection method for phase retrieval," vol. 20, no. 6, pp. 1025-1034, 2003. [Online]. Available: http://dx.doi.org/10.1364/josaa. 20.001025

[9] D. R. Luke, "Relaxed averaged alternating reflections for diffraction imaging," vol. 21, no. 1, pp. 37-50, nov 2004. [Online]. Available: http://dx.doi.org/10.1088/0266-5611/21/1/004

[10] J. Miao, J. Kirz, and D. Sayre, "The oversampling phasing method," vol. 56, no. 10, pp. 1312-1315, oct 2000. [Online]. Available: http://dx.doi.org/10.1107/s0907444900008970

[11] J. R. Fienup, "Reconstruction of a complex-valued object from the modulus of its fourier transform using a support constraint," vol. 4, no. 1, pp. 118-123, jan 1987. [Online]. Available: http: //dx.doi.org/10.1364/josaa.4.000118

[12] H. H. Bauschke, P. L. Combettes, and D. R. Luke, "Phase retrieval, error reduction algorithm, and fienup variants: A view from convex optimization,” vol. 19, no. 7, pp. 1334-1345, 2002. [Online]. Available: http://dx.doi.org/10.1364/josaa.19.001334

[13] E. J. Candès, Y. C. Eldar, T. Strohmer, and V. Voroninski, "Phase retrieval via matrix completion," vol. 57, no. 2, pp. 225-251, jan 2015. [Online]. Available: http://dx.doi.org/10.1137/151005099

[14] K. Jaganathan, Y. C. Eldar, and B. Hassibi, "Phase retrieval: An overview of recent developments," Nov 2015. [Online]. Available: http://arxiv.org/abs/1510.07713v1

[15] E. J. Candès, T. Strohmer, and V. Voroninski, "PhaseLift: Exact and stable signal recovery from magnitude measurements via convex programming," vol. 66, no. 8, pp. 1241-1274, nov 2012. [Online]. Available: http://dx.doi.org/10.1002/cpa.21432

[16] I. Waldspurger, A. dAspremont, and S. Mallat, "Phase recovery, maxcut and complex semidefinite programming," vol. 149, no. 1-2, pp. 47-81, dec 2015. [Online]. Available: http://dx.doi.org/10.1007/ s10107-013-0738-9

[17] F. Fogel, I. Waldspurger, and A. d'Aspremont, "Phase retrieval for imaging problems," Apr 2013. [Online]. Available: http://arxiv.org/abs/ $1304.7735 \mathrm{v} 2$

[18] E. J. Candès, X. Li, and M. Soltanolkotabi, "Phase retrieval from coded diffraction patterns," vol. 39, no. 2, pp. 277-299, oct 2015. [Online]. Available: http://dx.doi.org/10.1016/j.acha.2014.09.004

[19] D. Gross, F. Krahmer, and R. Kueng, "Improved recovery guarantees for phase retrieval from coded diffraction patterns," p. to appear, jul 2015. [Online]. Available: http://dx.doi.org/10.1016/j.acha.2015.05.004

[20] E. J. Candès, X. Li, and M. Soltanolkotabi, "Phase retrieval via wirtinger flow: Theory and algorithms," vol. 61, no. 4, pp. 1985-2007, apr 2015. [Online]. Available: http://dx.doi.org/10.1109/tit.2015.2399924

[21] J. Sun, Q. Qu, and J. Wright, "A geometric analysis of phase retrieval," Feb 2016. [Online]. Available: http://arxiv.org/abs/1602.06664v1

[22] K. Kreutz-Delgado, "The complex gradient operator and the cr-calculus," Jun 2009. [Online]. Available: http://arxiv.org/abs/0906.4835v1

[23] T. Steihaug, "The conjugate gradient method and trust regions in large scale optimization," vol. 20, no. 3, pp. 626-637, jun 1983. [Online]. Available: http://dx.doi.org/10.1137/0720042 
[24] J. Nocedal and S. Wright, Numerical Optimization. Springer Science \& Business Media, 2006.

[25] J. Sun, Q. Qu, and J. Wright, "When are nonconvex problems not scary?" Nov 2015. [Online]. Available: http://arxiv.org/abs/1510.06096v1

[26] P. Netrapalli, P. Jain, and S. Sanghavi, "Phase retrieval using alternating minimization," vol. 63, no. 18, pp. 4814-4826, sep 2015. [Online]. Available: http://dx.doi.org/10.1109/tsp.2015.2448516

[27] Y.-H. Dai, "Convergence properties of the bfgs algoritm," SIAM Journal on Optimization, vol. 13, no. 3, pp. 693-701, 2002.

[28] Y. Chen and E. J. Candes, "Solving random quadratic systems of equations is nearly as easy as solving linear systems," May 2015. [Online]. Available: http://arxiv.org/abs/1505.05114v1

[29] X. Li and V. Voroninski, "Sparse signal recovery from quadratic measurements via convex programming," vol. 45, no. 5, pp. 3019-3033, sep 2013. [Online]. Available: http://dx.doi.org/10.1137/120893707

[30] C. D. White, S. Sanghavi, and R. Ward, "The local convexity of solving systems of quadratic equations," Jun 2015. [Online]. Available: http://arxiv.org/abs/1506.07868v4 